\title{
Radar phase offset estimate using ionospheric field-aligned irregularities and aircraft
}

\author{
Ting-Han $\mathrm{Lin}^{1}$, Yen-Hsyang $\mathrm{Chu}^{1}$, Ching-Lun $\mathrm{Su}^{1, *}$, and Kuo-Feng Yang ${ }^{2}$ \\ ${ }^{1}$ Graduate Institute of Space Science, National Central University, Taoyuan City, Taiwan \\ ${ }^{2}$ National Chung-Shan Institute of Science and Technology, Taoyuan City, Taiwan
}

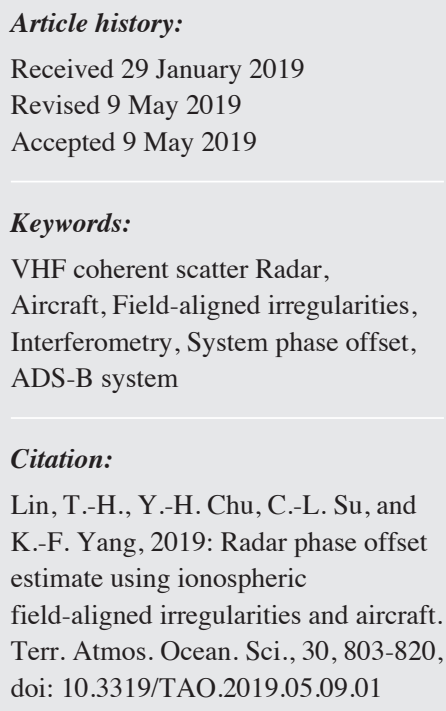

\begin{abstract}
The phase imbalance between receiving channels of a phase array antenna, referred to as phase offset, is one of the most crucial parameters in positioning the targets in lower and upper atmospheres using spatial domain interferometry (SDI) technique. In this study, we develop a method of using commercial aircraft that equips with Automatic Dependent Surveillance-Broadcast (ADS-B) system to estimate the system phase offsets of the Chungli VHF radar. The aviation data broadcasted from ADS-B system combined with the radar returns from the aircraft can obtain the system phase offset. The principle of the method and the algorithms of processing the ADS-B messages are described and the procedures of analyzing the aircraft echoes are also detailed in this article. On the basis of this method, multirotor equipped with a high precision GPS receiver is also employed to estimate radar system phase offset. The results show that the system phase offsets estimated by the aircraft and those from the multirotor are consistent. However, the aircraft/multirotor-derived system phase offsets are very different from those estimated from the radar returns of the 3-m ionospheric field-aligned plasma irregularities (FAIs) combined with International Geomagnetic Reference Field 12th generation (IGRF-12) model. The principle of the FAI method is introduced and the plausible causes of the discrepancies in the estimated system phase offsets between aircraft/multirotor and FAIs are discussed.
\end{abstract}

\section{INTRODUCTION}

Coherent scatter radar is one of the most important atmospheric remote sensing instruments that can detect echoes from refractive index irregularities in lower and upper atmospheres through Bragg volume scatter or Fresnel layer reflection (Balsley and Gage 1980). The atmospheric refractivity irregularities are primarily caused by the spatial random fluctuations of temperature, water vapor content, and ionospheric free electron density in the radar illuminating volume. In addition, hydrometeors and meteoric plasma in troposphere and mesosphere can also serve as the targets for the radars operating at frequencies higher than and lower than VHF band, respectively (Chu et al. 1991; Rapp and Lübken 2004; Li et al. 2014; Su et al. 2014). If the radar targets are present at a localized region in the illuminating volume, their positions can be determined from the phase differences of the radar returns received by a multi-baseline antenna system. In this context,

\footnotetext{
* Corresponding author

E-mail:clsu@jupiter.ss.ncu.edu.tw
}

accurate calibration of the system phase bias is required such that the system phase biases can be removed from the observed phase differences between difference receiving channel pairs to estimate true target position using spatial domain interferometry (SDI) technique.

Interferometry technique has long been applied to the coherent scatter radar to position and investigate ionospheric plasma irregularities for more than 3 decades. Notice that the ionospheric plasma irregularities at scale size of around $3-\mathrm{m}$ in $\mathrm{E}$ and $\mathrm{F}$ regions are characterized by the fieldaligned property, which are generated primarily from large scale plasma waves excited by plasma instability through non-linear energy cascade process (Farley 1985). That is, the distribution of the electron density along the magnetic field line is nearly uniform owing to extremely infrequent collision between free electron and neutral particle and that across the magnetic field line is highly randomly fluctuated because of the plasma turbulence cascade process. As a result, it is expected that the wavenumber power spectrum 
parallel to the magnetic field line for the former will have a very narrow width and peak at around $k_{\|}=0$, while the wavenumber power spectrum normal to the field line for the latter will be characterized by a very broad width and contains a specific Bragg wavenumber $k_{\perp}=k_{B}$ responsible for the backscatter of the incident radar wave, where $k_{\perp}$ and $k_{\|}$ are, respectively, the perpendicular and parallel wavenumbers with respect to the magnetic field line. Although the electron density perturbations of ionospheric field-aligned plasma irregularities (FAIs) in the parallel direction is so weak that magnetic field line can be treated as an equipotential line, there may exit very small wavenumbers in the parallel electron density fluctuations with $k_{\|}<<k_{\perp}$ to satisfy the relation $\overrightarrow{k_{B}}=\overrightarrow{k_{\perp}}+\overrightarrow{k_{\|}}$that is required for the Bragg backscatter. The ratio of $k_{\|}$to $k_{\perp}$ represents the incident angle of the radar wave vector deviated from the perpendicularity direction to the local magnetic field line and this angle is referred to as aspect angle. Because $k_{\|} \sim 0$, the aspect angle of the FAI echoes is expected to be very small, around $0.2^{\circ}$ (Wang et al. 2011). With this field-aligned property, the ionospheric plasma irregularities can thus be positioned by using interferometry technique implemented at a radar with multi-baseline antenna array. Nevertheless, the discrete targets without the field-aligned property can also be positioned by using spatial domain interferometry technique.

The use of interferometry technique to observe and study ionospheric plasma irregularities has been widely performed in the ionospheric community. Farley et al. (1981) first developed interferometry technique to position FAIs associated with equatorial electrojet in sporadic E (Es) region over Jicamarca Radio Observatory. On the basis of the same technique, Providakes et al. (1983) investigated the dynamic behavior of the ionospheric plasma irregularities occurred in auroral zone. Chu and Wang (1997) established interferometry capability at Chungli VHF radar to reconstruct 3-dimensional spatial structure of the 3-m FAIs responsible for the quasi-periodic (QP) echoes in Es region and found that the striated QP echoes are attributed to the isolated and patch-like plasma structures that repeatedly occurred and drifted horizontally in the east-west direction in the radar echoing region. Saito et al. (2007) used interferometry technique implemented on the MU radar to study the association of the QP echoes from 3-m FAIs in Es region with medium-scale traveling ionospheric disturbances (MSTID) in F region. Su et al. (2014) set up an interferometry antenna array at the Chungli VHF radar to position the meteor trails for the measurement of the semi-diurnal tide in mesosphere and lower thermosphere over the Chungli radar station. In addition to the need for the use of SDI technique to position ionospheric FAIs and meteor trails, precise calibration of the radar system phase bias is also indispensable to the radar experiment for the determination of the echo center location of the diffusive targets of the atmospheric refractivity fluctuations in lower atmosphere by using co- herent radar imaging and/or range imaging techniques (Yu and Palmer 2001; Chen et al. 2009, 2016; Tsai et al. 2018).

A phase imbalance between receiving channels refers to as phase offset, or systematic phase error, or initial system phase bias. Much work has been performed to estimate the phase offset using different methods, e.g., generating a common signal that simultaneously fed each of two receivers and measuring the phase offset between them (Aso et al. 1979; Vandepeer and Reid 1995; Valentic et al. 1997), calculating the trajectory of an airplane flying routinely in the vicinity of the radar and comparing the observed phase differences of the radar echoes between difference receiving channels with the optical-recorded ones (Robertson et al. 1953; Chen et al. 2002), utilizing single or a set of artificial radio beacons placed at known locations in the far-field of the radar array and measuring the phase present at each channel (Glanz 1971; Valentic et al. 1997; Chau et al. 2008), receiving beacon signals transmitted by satellites and radio stars which transit across receiving arrays, then calculating their trajectories and comparing the phase differences of the beacon signals between difference channels with the theoretical ones (Glanz 1971; Clark 1978; Palmer et al. 1996; Sullivan et al. 2006; Chau et al. 2008, 2014; Schlatter et al. 2013), using meteor-head or -trail echoes collected during routine observations to do the self-survey phase calibration (Valentic et al. 1997; Holdsworth et al. 2004; Chau et al. 2008), or comparing the distribution of sporadic E echoes with the expected echoing region which is determined in accordance with the International Geomagnetic Reference Field (IGRF) model (Wang 1999; Wang and Chu 2001; Kuong et al. 2003).

It is noteworthy that the above-mentioned methods are subject to respective drawbacks, leading to estimate uncertainty of the system phase offset. For example, the approach of feeding a common signal to receivers can only estimate the phase offset due to the aging of hardware components, or imperfections inside receivers. However, the offset caused by displacement of the center of the antenna array cannot be calibrated by using this method (Valentic et al. 1997). The satellite/radio star method can only be performed on a special day when they transit over the radar beam in a very quiet condition without the presence of ionospheric irregularities that may cause signal scintillation (Chau et al.2008). The radar phase offset estimated by using the IGRF method is susceptible to the inhomogeneous distribution of the 3-m FAIs in the expected echoing region, leading to uncertainty of the estimated phase offset.

An attempt was made in this study to develop an approach that employs commercial aircraft as a radio source, which equips with automatic dependent surveillance-broadcast (ADS-B) avionics system, to estimate phase offsets of the ionospheric array of Chungli VHF radar from the received ADS-B signals. ADS-B is a surveillance technique which is widely used around the world for air traffic control, 
which can broadcast aircraft flight identification, position, ground velocity, barometric or geometric altitude, vertical climb and descent rates, track angle, emergency indication, and other information at $1090 \mathrm{MHz}$ (ICAO 2008). From the broadcasted messages of the ADS-B avionics system, the 3-dimensional trajectory of the aircraft can be reconstructed and the corresponding phase differences between different receiving channels can thus be computed. In addition to the use of the broadcast messages, the phase differences can also be measured from the radar returns backscattered from the aircraft, in which the radar system phase offsets are included. By comparing radar-measured and ADS-B-determined phase differences, we can deduce the radar system phase offsets of the difference receiving channel pairs.

This paper is organized as follows. Section 2 describes the methods of using aircraft equipped with ADS-B system and the ionospheric 3-m FAIs combined with International Geomagnetic Reference Field 12th generation (IGRF-12) model to estimate radar system phase offsets. The key components of the aircraft method, such as coordinate transform from World Geodetic System 1984 (WGS84) to transverse Mercator projection in two-degree zones (TM2), aircraft trajectory reconstruction from ADS-B messages and time synchronization between radar and aircraft will be elaborated in Appendix 1, 2, and 3, respectively. Section 3 presents the results of the estimated system phase offsets using different methods. Discussions will be given in section 4 and conclusion will be drawn in section 5 .

\section{METHODOLOGY}

The objective of the present study is to use the radar returns from various targets, including ionospheric FAIs, commercial aircraft equipped with ADS-B avionics system, and unmanned radio-controlled multirotor equipped with high precision GPS receiver, to estimate the system phase offsets of the ionospheric receiving channels of the Chungli VHF radar. Figure 1 displays the ionospheric antenna array of the Chungli VHF radar, which is designed and operated exclusively for the observation and investigation of the 3-m FAIs in $\mathrm{E}$ and $\mathrm{F}$ regions. As shown, the antenna array consists of three independent and identical subarrays A, $\mathrm{B}$, and $\mathrm{C}$ arranged as an isosceles triangle in shape and are connected to respective transmitters and receivers for radar wave transmission and reception. The central positions of the subarrays that were determined by a Trimble R6 GNSS system are given below:

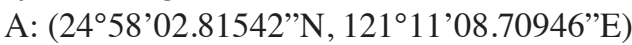

B: $\left(24^{\circ} 58^{\prime} 03.82412^{\prime \prime} \mathrm{N}, 121^{\circ} 11^{\prime} 07.98267^{\prime \prime} \mathrm{E}\right)$

C: (24⒌ $\left.58^{\prime} 04.01582^{\prime \prime} \mathrm{N}, 121^{\circ} 11^{\prime} 08.59037^{\prime \prime} \mathrm{E}\right)$

Note that the maximum errors of the positions determined by the Trimble R6 GNSS system is approximately $3 \mathrm{~mm}$ in horizontal and $3.5 \mathrm{~mm}$ in vertical directions. Each subarray comprises $32(4 \times 8)$ linearly polarized 4-element Yagi an- tennas. The operation frequency of the Chungli VHF radar is $52 \mathrm{MHz}$, corresponding to a wavelength of $5.77 \mathrm{~m}$. The half-power beam width of the two-way transmitting pattern of each subarray is $7.8^{\circ}$ in elevation and $18^{\circ}$ in azimuth with a boresight pointed in a direction of $19.16^{\circ}$ west of geographic north in azimuth and $50.2^{\circ}$ in elevation. For the present study, the subarray A was used to transmit the radar wave and all of the three subarrays were used for receiving the radar returns.

\subsection{Ionospheric FAI Method}

As mentioned before, the echoing region of ionospheric FAIs comprises the points of the radar wave vectors nearly perpendicular to the magnetic field lines. The angle made by the wave vector of the radar return from FAIs and the perpendicularity to the magnetic field line is referred to as aspect angle. Radar experiments reveal that the aspect angles of the FAI echoes in $\mathrm{E}$ and $\mathrm{F}$ regions are very small, primarily less than $0.2^{\circ}$ (Wang et al. 2011). With the help of IGRF-12 model that provides the 3-dimensional configuration of magnetic field lines over the ground-based radar station, the echoing region with a given aspect angle relative to the perpendicularity to the local magnetic field lines can be accurately determined. This IGRF-12-determined echoing region is referred to as expected echoing region in this manuscript, which is a unique echoing region for a groundbased radar and only the echoes scattered from the FAIs

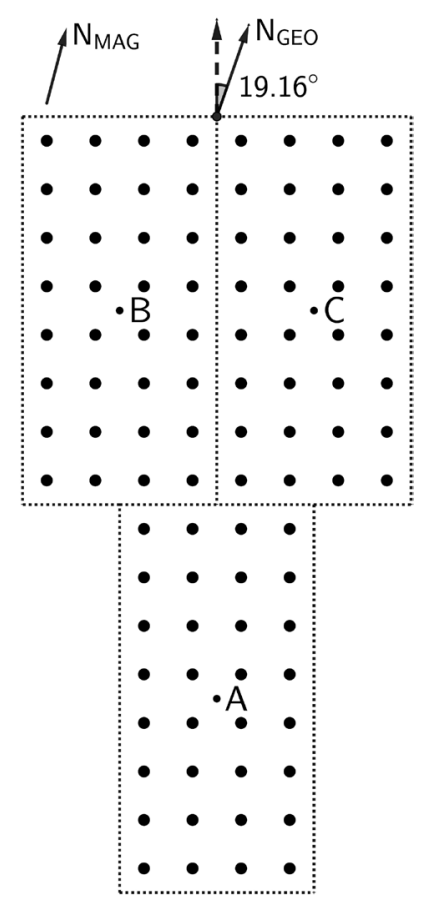

Fig. 1. Schematic diagram of the ionospheric antenna array of the Chungli VHF radar, which is composed of three independent and identical subarrays. The arrows marked with $\mathrm{N}_{\mathrm{GEO}}$ and $\mathrm{N}_{\mathrm{MAG}}$ represent the directions of geographic north and magnetic north, respectively. 
present in this region can be detected by the radar. In light of the fact that the measured phase differences between different receiving channels contain the system phase bias, a comparison of the radar-measured phase differences and IGRF-12-calculated phase differences can deduce the radar system phase offsets (Chu and Wang 1997; Wang 1999; Lin et al. 2016). Figure 2 shows its expected echoing regions for the years of 1986 and 2018, in which the FAI height range of $100-110 \mathrm{~km}$ and aspect angle of $0.1^{\circ}$ are given to calculate the echoing region from IGRF-12 model. A Monte Carlo method is used to generate the locations of the FAIs in the expected echoing region, in which a 2-dimensional Gaussian probability density function that is best fitted to the antenna beam pattern is assumed. As shown, the expected echoing region predicted by IGRF-12 model is shifted over time due to the temporal drift of the geomagnetic field.

The locations of the 3-m FAIs in the echoing region can be identified and positioned from their echoes by using interferometry technique. The elevation angle $\theta$ and azimuth angle $\phi$ can be estimated from the phase differences of the FAI echoes detected by different receiving channel pairs in accordance with following equations (Chu and Wang 1997; Lin et al. 2016)

$\phi=\cot ^{-1}\left[\tan \beta+\frac{d_{C B}}{d_{B A} \cos \beta} \frac{\left(\Phi_{B A}+2 \pi L+\Delta \Phi_{B A}\right)}{\left(\Phi_{C B}+2 \pi M+\Delta \Phi_{C B}\right)}\right]$

$\theta=\cos ^{-1}\left(\frac{\Delta \Phi_{C B}+2 \pi M}{k d_{C B} \sin \phi}\right)$

where $k=2 \pi / \lambda$ is radar wavenumber and $\lambda$ is wavelength, $\beta \approx 14^{\circ}, L$ and $M$ are interferometry lobe numbers that are 0 and 4 for the antenna pairs B-A and C-B, respectively, $d_{C B}$ $=18 \mathrm{~m}$ and $d_{B A} \approx 37.1 \mathrm{~m}$ are, respectively, the lengths of the baselines B-C and B-A, $\Phi_{B A}$ and $\Phi_{C B}$ are, respectively, the phase differences of the radar returns between antenna array pairs B-A and C-B. If the estimated angles $\theta$ and $\phi$ from the observed FAI echoes are not consist with the expected angles calculated from IGRF-12 model, there are system phase biases in the observed radar returns. A comparison between observed and expected echoing regions of the FAIs can obtain the system phase biases of the different receiving channel pairs. Figure 3 shows an example of the comparison results for the data collected for the period from 1 July to 28 July 2017. As indicated, there is a slightly offset between observed (left panel) and calibrated (right panel) echoing regions, which represent the system phase biases for different antenna subarray pairs. In this study, we compute the 2-dimensional cross correlation function between radarmeasured FAI echoing region and IGRF-12-predicted echoing region to estimate the phase offsets. The result reveals that the values of the phase biases $\Delta \Phi_{C B}, \Delta \Phi_{B A}, \Delta \Phi_{C A}$ are $+13^{\circ},-4^{\circ}$, and $+9^{\circ}$, respectively.

\subsection{Commercial Aircraft and Multirotor Method}

\subsubsection{ADS-B System and Signal Processing}

In addition to the ionospheric FAIs, the commercial aircraft equipped with ADS-B system and multirotor equipped with highly accurate GPS receiver can also be employed to calibrate the radar system phase bias in accordance with the following procedure. To begin with, the phase differences between different pairs of the receiving channels and radial velocities of the aircraft or multirotor are calculated first. Secondly, the signals containing the information on the position and velocity of the aircraft or multirotor, which are broadcasted by the ADS-B system or obtained from the GPS facility, are received and decoded by the collocated ground receiver. Thirdly, the GPS position of the aircraft is transferred to a conformal map projection coordinate. Fourthly, the trajectory of the aircraft or multirotor was retrieved and synchronized with the radar echoes. Finally, system phase offsets of receiving channels are estimated.

The aircraft experiment was conducted during three periods, namely, 23 August 2018 to 3 September 2018, 23 March 2018 to 8 April 2018, and 20 December 2018 to 6 January 2019. The radar returns from the commercial aircraft when they transited across the main antenna pattern of the ionospheric array of Chungli VHF radar were collected. The radar parameters set for the radar experiment are given below. The pulse repetition frequency was $4000 \mathrm{~Hz}$ and 4 times of coherent integration was made, corresponding to a time resolution of $1 \mathrm{~ms}$; the extent of observing range was from $450 \mathrm{~m}$ to $21.9 \mathrm{~km}$; the pulse length was $1 \mu$ s without any phase coding, which corresponds to a range resolution of $150 \mathrm{~m}$.

The amplitude of the radar returns from aircraft or multirotor received by two different antenna subarrays $A_{i}$ and $A_{k}$ can be simply expressed as

$S_{i}(t)=G_{i} e^{j\left[2 \pi f t+\Phi_{i}(t)+\Delta \Phi_{i}\right]}$

$S_{k}(t)=G_{k} e^{j\left[2 \pi f t+\Phi_{k}(t)+\Delta \Phi_{k}\right]}$

where $i, k=1,2,3$ represent subarrays A, B, and C, respectively. $G_{i}, G_{k}$ represent corresponding amplitudes, $\Phi_{i}(t)$, $\Phi_{k}(t)$ represent the path phase of the radar waves detected by respective receiving channels, $\Delta \Phi_{i}, \Delta \Phi_{k}$ represent the radar system phase biases of corresponding channels. Therefore, the phase difference between two receiving channels can be expressed as

$\Phi_{i k}^{R}(t)=\Phi_{i k}(t)-2 \pi L_{i k}(t)+\Delta \Phi_{i k}$

where $\Phi_{i k}(t)=\Phi_{k}(t)-\Phi_{i}(t)$ is the true phase difference of the radar returns from target, $L_{i k}$ is a positive integer that corrects phase ambiguity. Considering the boresight 


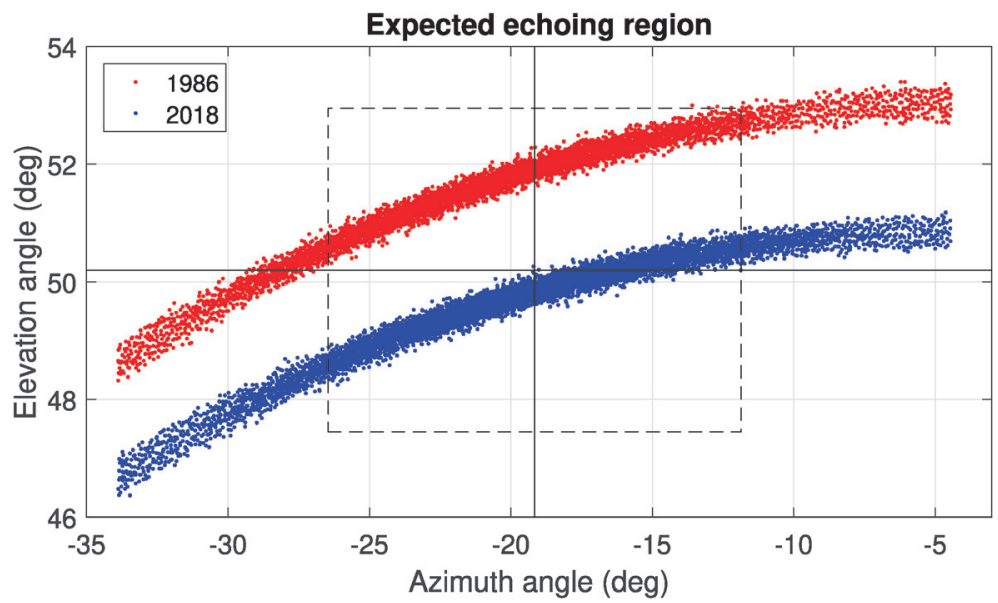

Fig. 2. Expected echoing regions of the 3-m ionospheric field-aligned irregularities for the Chungli VHF radar for 1986 and 2018 , in which the azimuth angle is relative to the geographic north. The dot densities represent the occurrence probability of the echoes of the FAIs that distribute uniformly in the echoing regions, which is a function of the gain of the antenna beam pattern.
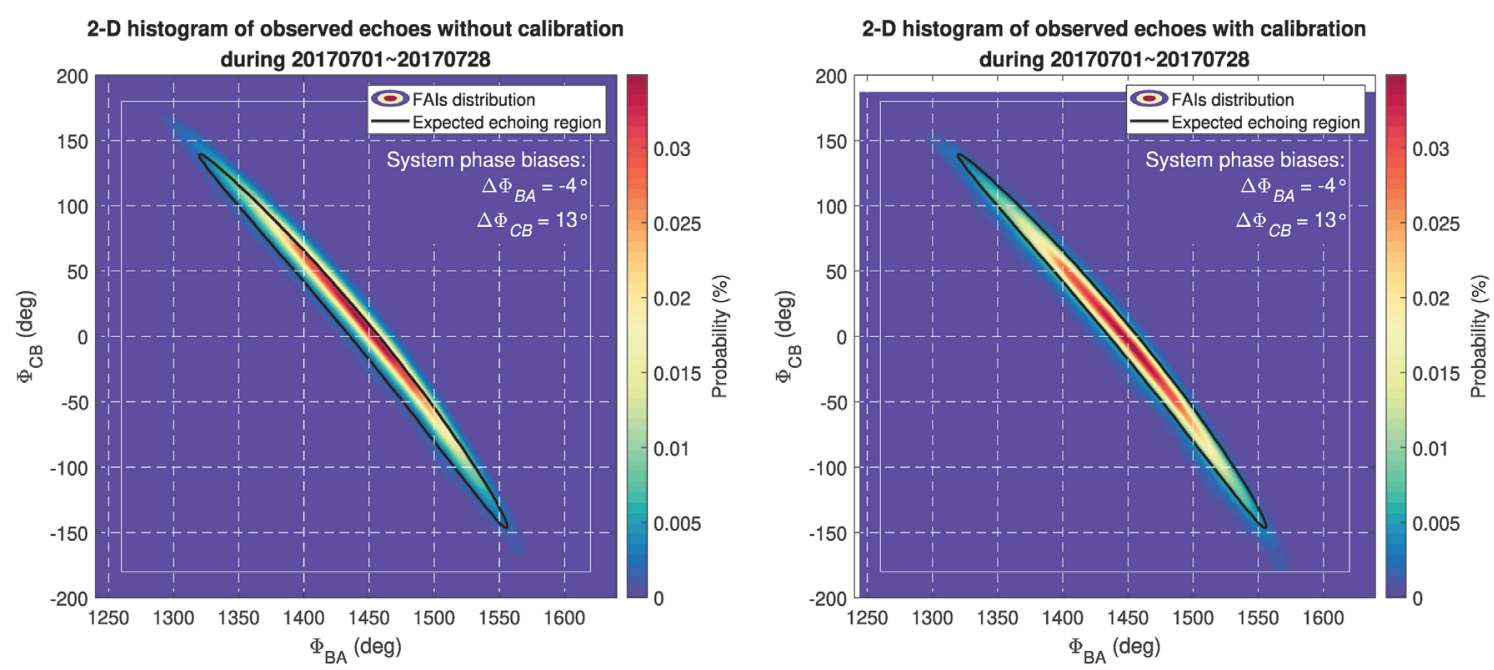

Fig. 3. Examples of comparing phase differences $\Delta \Phi_{C B}$ and $\Delta \Phi_{B A}$ of the observed FAI echoes and those of the expected echoing region for the period 1 July to 28 July 2017, in which the contours represent the occurrence frequencies of the observed FAI echoes and the areas encircled by the black curves are the expected echoing regions calculated from the IGRF-12 model. Left panel (right panel) shows the FAI contour before (after) phase adjustments of $-4^{\circ}$ in $\Delta \Phi_{B A}$ and $+13^{\circ}$ in $\Delta \Phi_{C B}$.

direction of the ionospheric array of the Chungli VHF radar, we find that the values of $L_{C B}, L_{B A}, L_{C A}$ are, respectively, 0,4 , 4 , which are consistent with the previous result (Wang and Chu 2001). $\Delta \Phi_{i k}=\Delta \Phi_{k}-\Delta \Phi_{i}$ are the phase offsets that we try to obtain in this study. Additionally, the Doppler (radial) velocity of the radar returns from target received by $i^{\text {th }}$ antenna subarray can be expressed as

$V_{A_{i} T}^{R}(t)=V_{i}^{R}(t)=\frac{\lambda}{4 \pi} \frac{d \Phi_{i}(t)}{d t}$

where $\lambda$ is the wavelength of the radar. Figure 4 shows a typical case of the range-time variation of normalized am- plitudes of radar echoes (lower panel). The radar echo is coming from the aircraft B-LCN on 30 August 2018. As depicted in lower panel of Fig. 4, the radar first received the aircraft echoes at approximately $17.85 \mathrm{~km}$ away from the radar at 18:56:41 LT, and about half minute later, the aircraft echo is lost at the range $15.75 \mathrm{~km}$. The corresponding phase differences of the aircraft echoes between the different antenna subarray pairs are shown in upper panel of Fig. 4. As shown, the observed phase difference $\Phi_{C B}^{R}$ is approximately $0^{\circ}$ at 18:56:58 LT, and $\Phi_{B A}^{R}$ is equal to $\Phi_{C A}^{R}$ at the same time. In light of the fact that the observed phase differences $\Phi_{C B}^{R}, \Phi_{B A}^{R}$, and $\Phi_{C A}^{R}$ were not calibrated, these features cannot be considered that the aircraft was transiting across the boresight of antenna beam at the time. 
(a) Trend of observed phase differences on 2018-08-30

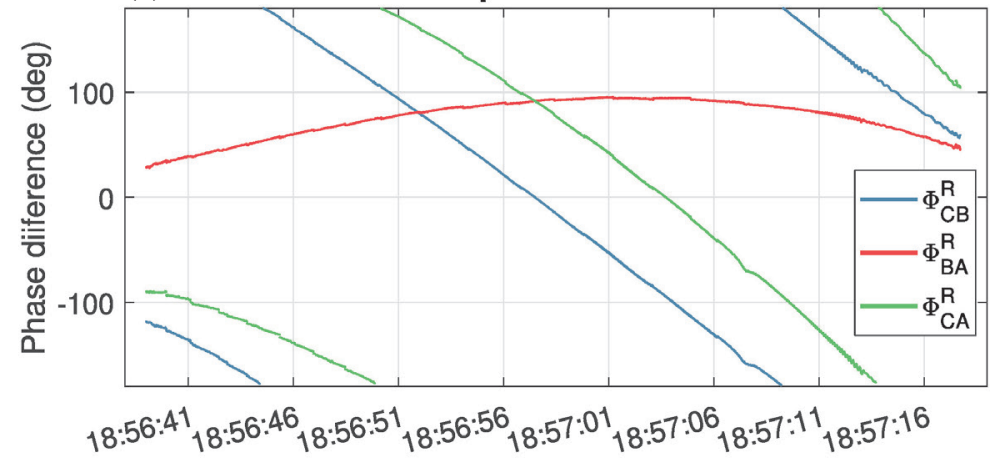

(b) Trend of normalized echo amplitude in range gates

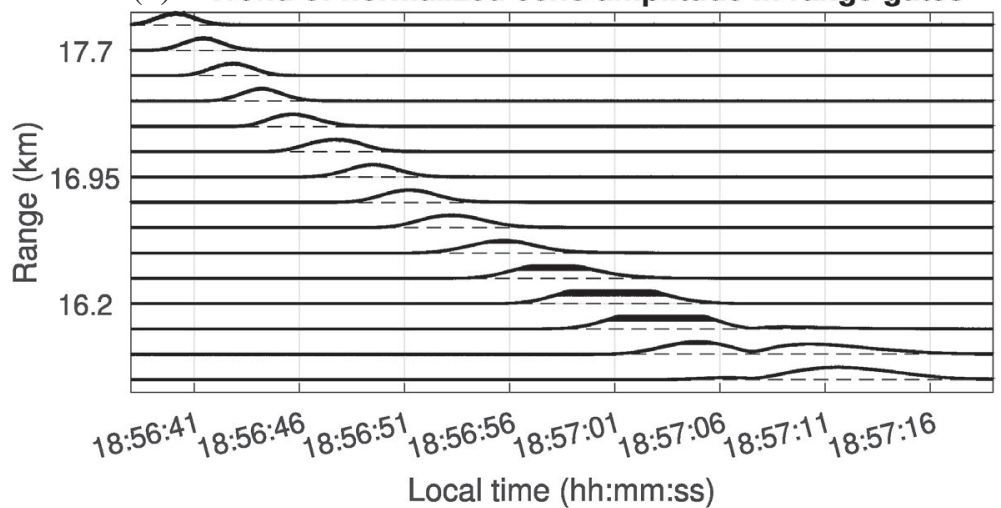

Fig. 4. Phase differences (upper panel) of the aircraft echoes (lower panel) detected by the Chungli VHF radar from the aircraft B-LCN on 30 August 2018. Dashed-horizontal lines in lower panel separate range gates and the solid line represents the normalized echo amplitude as a function of time.

In addition to the radar experiments, we utilize commercial Digital Video Broadcast Terrestrial (DVB-T) USB dongle with a RTL2832U demodulator chipset and a R820T2 tuner chip to develop a receiver to record and decode ADS-B 1090ES messages. A handmade coaxial collinear antenna made from RG58 cables was designed and placed on the roof of Chungli VHF radar station, which is approximately $50 \mathrm{~m}$ away from the ionospheric array. Moreover, with the Communications Toolbox Support Package for RTL-SDR Radio in MATLAB functions, we process the ADS-B signals to acquire the aircraft messages in nearly real time (Stewart et al. 2015). Note that only the position and speed information were utilized to reconstruct the trajectory of aircraft in this experiment.

According to the OpenSky research sensor network in Central Europe, approximately two-thirds of all aircraft that crossed the receiving range of the sensor network broadcast ADS-B messages (Strohmeier et al. 2014). Moreover, as of December 2012, Vidal (2013) reported that more than 81 percent of all AIRBUS commercial aircraft were already equipped with ADS-B transponders. Both statistics indicate that the global coverage of the ADS-B signal is high.

In the vicinity of Chungli VHF radar station, the airtraffic density is very high because two airports are located in this region. One is the Taoyuan International Airport
(TPE) located approximately $15 \mathrm{~km}$ north-northeast of the radar station, and the other one is Taipei Songshan Airport (TSA) located approximately $40 \mathrm{~km}$ east-northeast of the radar station. Furthermore, two regular international air routes, namely A1 and M750, also cross through this region and connect most of metropolises in East Asia. Based on the 1090ES messages received by the DVB-T USB dongle, the average air-traffic density around Chungli VHF radar station is very high, about 60 aircraft per hour. However, it has been shown that a denser air traffic density leads to a higher error rate for the ADS-B packet because of the message collision effect (Strohmeier et al. 2014). In other words, frequent failures may occur in the decoding of 1090ES messages in dense air spaces, which leads to the unavailability of the position and velocity information of aircraft. According to the record of our 1090ES receiving system, the decoding of messages had a fail rate of 30 percent during the whole experiment. Fortunately, for an aircraft flying in a steady state without changing the direction of heading, speed, and height, it is still possible to reconstruct the continuous trajectory of the aircraft based on the discontinuous position and velocity data. Therefore, only those aircraft flying in a steady state were chosen as the observation targets in this experiment.

Figure 5 shows 13 tracks of aircraft that flew directly 


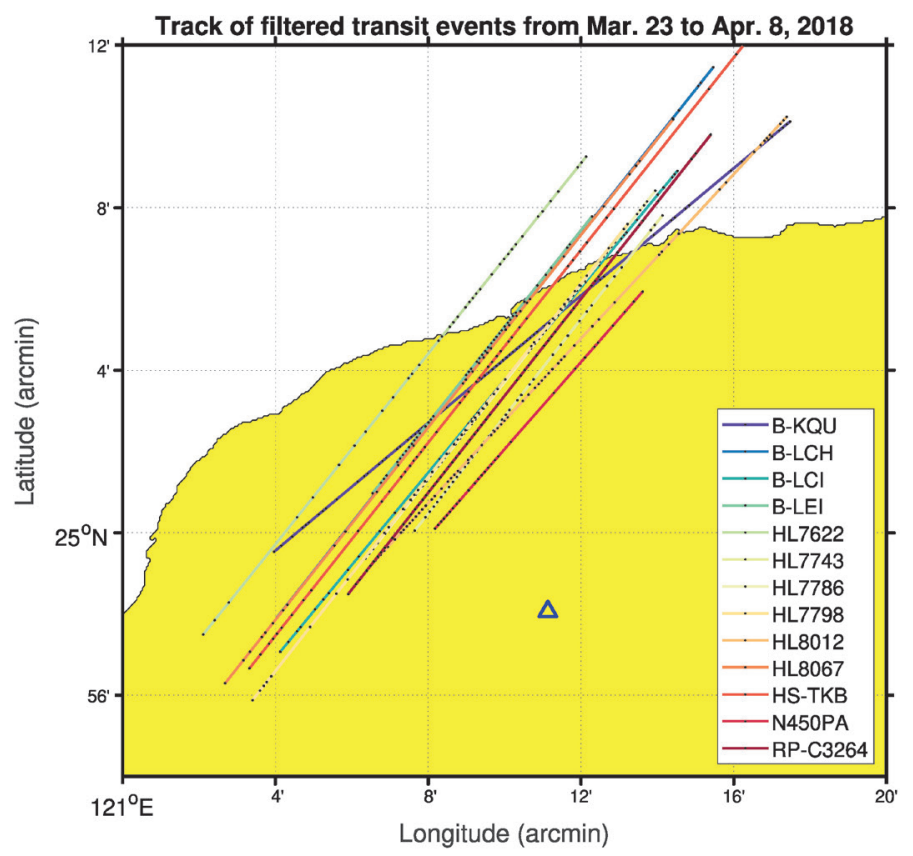

Fig. 5. Example of the trajectories of commercial aircraft equipped with ADS-B system that flew across the radar beam of the ionospheric array of the Chungli VHF radar for the periods from 23 March 2018 to 8 April 2018. The blue triangle represents the location of Chungli VHF radar station, uneven black dots represent broadcasted horizontal positions of each flight in the WGS84 coordinate system, and the lines with different colors represent the regressive lines best fitted to the tracks of the corresponding aircraft.

across the main antenna beam of the ionospheric array of the Chungli VHF radar for the period from 23 March 2018 to 8 April 2018. The blue triangle represents the location of Chungli VHF radar station, uneven black dots represent broadcasted horizontal positions of each flight in the WGS84 coordinate system, colored lines represent the regression lines best fitted to the tracks of the corresponding aircraft. It should be noted that these aircraft all maintain at their respective steady altitudes and speeds during their passages through the main antenna beam. In addition, on the basis of additional ADS-B position accuracy information received during the same period, all broadcasted positions have a horizontal error less than $30 \mathrm{~m}$ with 95 percent probability and vertical error less than $45 \mathrm{~m}$ with 95 percent probability. The uncertainty based on the position error will be discussed in section 4 .

\subsubsection{Coordinate Transformation}

The coordinate system that determines the commercial aircraft positions from the message broadcasted by the ADSB system is the geographic coordinate WGS84 that bases on an oblate spheroid with equatorial radius of $6378.137 \mathrm{~km}$ and polar radius of $6356.752 \mathrm{~km}$. Therefore, the longitude, latitude and altitude specified a point located in WGS84 are different from those in a coordinate system based on a perfect sphere. This global coordinate is not suitable for calculating accurate bearings and distances employed for the estimate of the radar system phase bias. It should be noted that the absolute bearings and distance between the subarray center and the aircraft are the most crucial factors influencing the observed phase difference between receiving channels. In other to calculate the bearings and distance with great accuracy, the TM2 projection coordinate was utilized in this experiment. The transverse Mercator projection is a conformal map projection, which is preserving angles and shapes of objects in small areas. The details of the coordinate transform from the geographic coordinate WGS84 to the Mercator coordinate will be given in Appendix 1.

\subsubsection{Aircraft Trajectory Reconstruction}

Two difficulties would raise when commercial aircraft were chosen to be the reference target. One is the issue of dense air traffic density around Chungli VHF radar, which leads to frequent fails of the decoding of 1090ES messages. The other one is time synchronization between radar and aircraft or multirotor, which is very critical in estimating phase offsets of the radar system. However, it is noteworthy that, on the basis of the protocol of 1090ES service, the time stamp of aircraft positions will not be broadcasted. Without the time stamp information, the time synchronization between radar echoes and ADS-B data is difficult to perform. Fortunately, from the information on speed and position of aircraft broadcasted by the ADS-B system, the trend of the radial velocity of aircraft can be obtained. By comparing 
the ADS-B broadcasted radial velocity of the aircraft with radar measured Doppler velocity of the aircraft echoes, the time difference between these two radial velocities can be estimated and performing the time synchronization is therefore possible. The aircraft trajectory reconstruction from the ADS-B messages to the Mercator coordinate and the time synchronization between aircraft and radar will be detailed in Appendix 2 and 3, respectively.

Assume $\Delta V_{T}=0 \mathrm{~m} \mathrm{~s}^{-1}, t_{0}=0 \mathrm{~s}$. The trajectory of the aircraft was reconstructed in accordance with Eqs. (A5a) and (A5b) from the ADS-B messages at $1 \mathrm{~ms}$ time resolution for a total duration of $285 \mathrm{~s}$. Figure 6a presents an example of the reconstructed trajectory of the aircraft B-LCN in TM2 coordinate. According to Eqs. (A7b) and (A7e), the phase differences between different antenna subarray pairs and the aircraft radial velocity with respect to subarray A were retrieved as shown the time sequences in Figs. $6 \mathrm{~b}$ and $\mathrm{c}$, respectively, in which the transit point is defined as the point that the aircraft flies across the boresight of the radar beam. The positive (negative) time represents the time when the aircraft was leaving (approaching) the transit point, and, therefore, positive (negative) radial velocity signifies the aircraft flying away (toward) the radar. Figure 6d illustrates the transiting path of the aircraft in azimuth-elevation plane, in which the azimuth angle of $-19.16^{\circ}$ represents the boresight direction and $0^{\circ}$ is the geographic north. The contour in Fig. 6d represents the two-way antenna beam pattern of the subarray A, the black solid line represents the path of the aircraft during the observation period, the yellow dashed elliptic curve marks the $-9 \mathrm{dBi}$ contour of the main beam pattern, and the white solid curve highlights the aircraft path inside the main beam. As shown in Fig. 6c, there is a radial velocity of about $-60 \mathrm{~m} \mathrm{~s}^{-1}$ when the aircraft flew across the transit point. This feature is the result of that fact that there is an angle of about $115^{\circ}$ between the aircraft flight direction and the antenna beam boresight direction, which combined with the aircraft velocity is responsible for the radial velocity. From Figs. 6a, b, c, and d, it is clear that the aircraft flew from northeast to southwest at a velocity ranging from -230 to $+100 \mathrm{~m} \mathrm{~s}^{-1}$. Figure $6 \mathrm{~b}$ reveals that, when the aircraft was transiting across the boresight of the radar, the phase difference $\Phi_{C B}$ was expected to be very close to $0^{\circ}$, and $\Phi_{B A}$ was anticipated to be equal to $\Phi_{C A}$. Note that in the whole experiment, only the data of 80 aircraft that flew across the main radar beam were collected for estimating system phase offsets of the radar.

\subsubsection{System Phase Offset Estimation}

As shown in Eq. (3), the radar-measured phase difference of the aircraft echoes between $i^{\text {th }}$ and $k^{\text {th }}$ antenna subarray pair $\Phi_{i k}^{R}(t)$ contains not only the true path phase difference $\Phi_{i k}(t)$ of the aircraft, but also the system phase offset $\Delta \Phi_{i k}$. Although exact value of $\Phi_{i k}(t)$ is unknown, we can use ADS-B broadcasted aircraft position to estimate the path phase difference $\Phi_{i k}^{*}(t)$ to approximate to $\Phi_{i k}(t)$ with acceptable reliability and accuracy based on ADS-B message. Because the integers for phase ambiguity corrections of $i^{\text {th }}$ and $k^{\text {th }}$ antenna subarrays $L_{i k}$ have been determined already at the Chungli VHF radar, the unwrapped (a) Track of the aircraft "B-LCN" on 2018-08-30, 18:56 LT

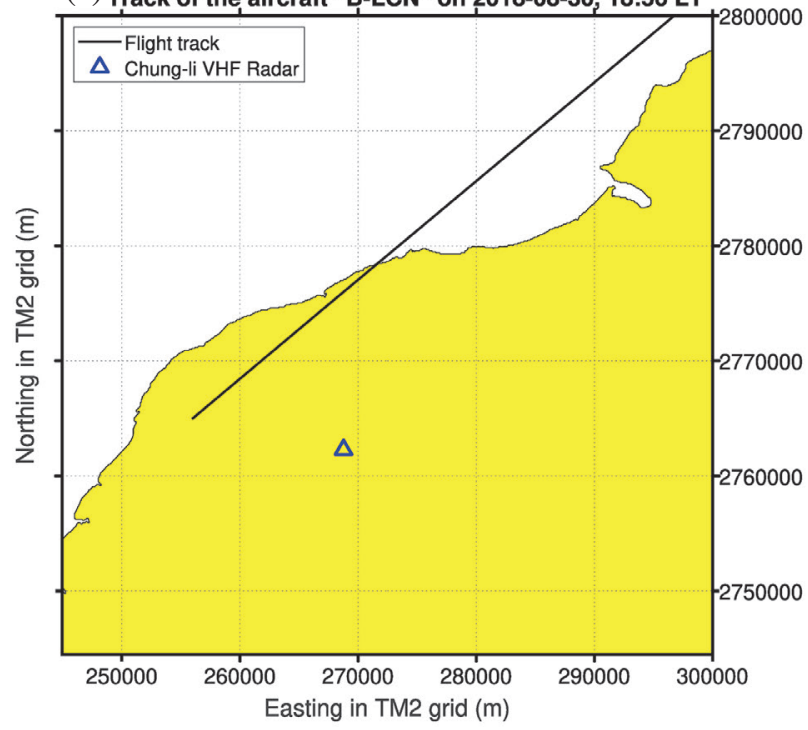

(b)

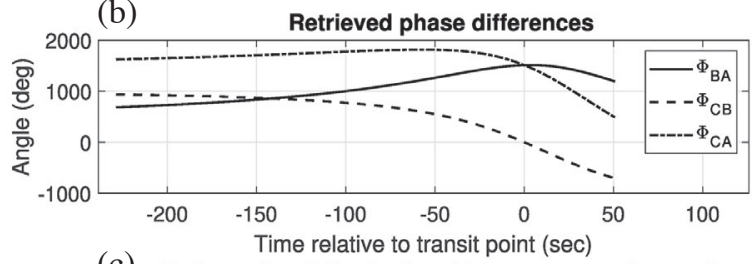

(c) Estimated radial velocity with respect to subarray $\mathbf{A}$
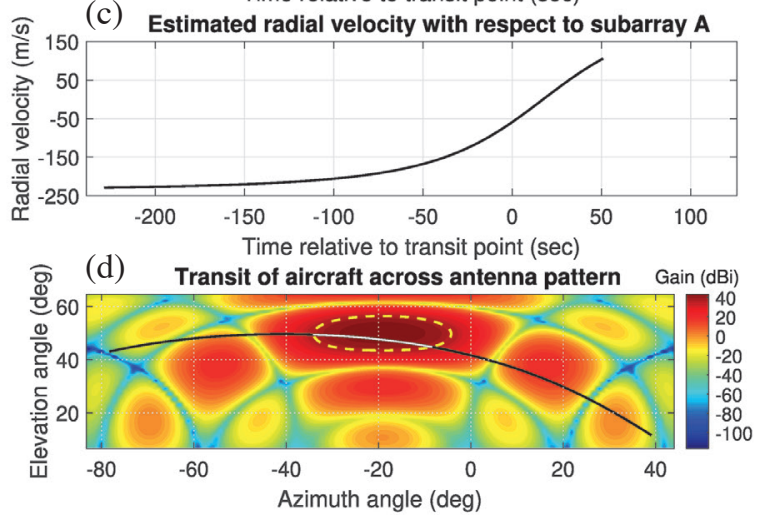

Fig. 6. (a) Projection of the track of the aircraft B-LCN in TM2 coordinate system. (b) Trend of retrieved phase difference pairs of the aircraft B$\mathrm{LCN}$, the $\mathrm{x}$-axis indicates the time relative to the transit point. (c) Trend of the estimated radial velocity with respect to subarray A during the same period in (b), a positive (negative) value of radial velocity indicates that the aircraft is flying away from (toward) the radar. (d) Path of the aircraft in azimuth-elevation plane, the filled contour illustrates the antenna pattern of the radar. 
radar-measured phase difference $\Phi_{i k}^{R U}(t)=\Phi_{i k}^{R}(t)+2 \pi L_{i k}(t)$ can thus be obtained. Considering the random fluctuations of the radar measurements, we estimate $\Delta \Phi_{i k}$ by taking average of the difference between $\Phi_{i k}^{R U}(t)$ and $\Phi_{i k}^{R}(t)$ over a time period. Namely,

$\Delta \Phi_{i k}=\overline{\Phi_{i k}^{R U}(t)-\Phi_{i k}^{*}(t)}$

In the case of the aircraft B-LCN, phase offsets $\Delta \Phi_{C B}$, $\Delta \Phi_{B A}$, and $\Delta \Phi_{C A}$ are $-6.87^{\circ},+21.54^{\circ}$, and $+14.67^{\circ}$, respectively, and the corresponding RMSE are $1.38^{\circ}, 1.10^{\circ}$, and $1.12^{\circ}$, respectively. Figure 7 shows the comparison between ADS-B-derived phase differences and radar-measured phase differences of the aircraft B-LCN. Black points represent the trend of unwrapped radar-measured phase difference, blue points represent the phase difference which is adjusted according to the corresponding phase offset, and the black solid line represents the synchronized ADS-Bderived phase difference as a reference. Obviously, all three adjusted radar-measured phase differences perfectly match the corresponding ADS-B-derived phase difference, small RMSE values also indicate that estimated phase offsets are all highly reliable.

\section{RESULTS}

Figure 8 shows the time series of the estimated system phase offsets for the period from 23 August 2018 to 3 September 2018, in which 50 aircraft transiting events were analyzed. The error bar of each event signifies the corresponding RMSE of the phase offset. As shown, the mean values of the system phase biases of $\Delta \Phi_{C B}, \Delta \Phi_{B A}$, and $\Delta \Phi_{C A}$ for this period are $-8.66^{\circ},+22.34^{\circ}$, and $+13.68^{\circ}$, respectively. The averages of RMSEs of $\Delta \Phi_{C B}, \Delta \Phi_{B A}$, and $\Delta \Phi_{C A}$ are $1.979^{\circ}, 1.911^{\circ}, 2.153^{\circ}$, respectively, which are approximately $2^{\circ}$ and comparable with the results reported by other radar groups in magnitude (Palmer et al. 1996; Valentic et al. 1997; Chen et al. 2002; Sullivan et al. 2006). The standard deviations of the estimated phase offsets are very stable over time with the values less than $3^{\circ}$, which are $1.79^{\circ}, 2.99^{\circ}$, and $2.59^{\circ}$ for $\Delta \Phi_{C B}, \Delta \Phi_{B A}$, and $\Delta \Phi_{C A}$, respectively, and consistent with other results in magnitude (Chen et al. 2002; Chau et al. 2008, 2014).

Figure 9 shows the estimated system phase offsets for the period from 23 March 2018 to 8 April 2018, all 13 aircraft transiting events were collected during this period. The mean values of the system phase biases of $\Delta \Phi_{C B}, \Delta \Phi_{B A}$, and $\Delta \Phi_{C A}$ for this period are $-7.26^{\circ},+19.7^{\circ}$, and $+12.45^{\circ}$, respectively. The averaged RMSE values of $\Delta \Phi_{C B}, \Delta \Phi_{B A}$, and $\Delta \Phi_{C A}$ during this period are $1.529^{\circ}, 2.057^{\circ}, 2.481^{\circ}$ and the standard deviations are $1.21^{\circ}, 4.0^{\circ}, 4.33^{\circ}$, respectively.

In addition to above results, the system phase biases are also estimated by using multirotor and ionospheric FAIs. Table 1 summarizes all of the experimental results. As shown, the estimated system phase biases made from the aircraft and multirotors are essentially very consistent. The means and the standards of the estimates for $\Delta \Phi_{C B}$, $\Delta \Phi_{B A}$, and $\Delta \Phi_{C A}$ are, respectively, in the ranges of $-5.10^{\circ}$ $\sim-8.66^{\circ},+19.7^{\circ} \sim+22.34^{\circ}$, and $+12.45^{\circ} \sim+16.59^{\circ}$ for the mean values and $1.21^{\circ} \sim 3.38^{\circ}, 2.54^{\circ} \sim 4.00^{\circ}, 2.11^{\circ} \sim 5.53^{\circ}$ for the standard deviations. The variation extents of the
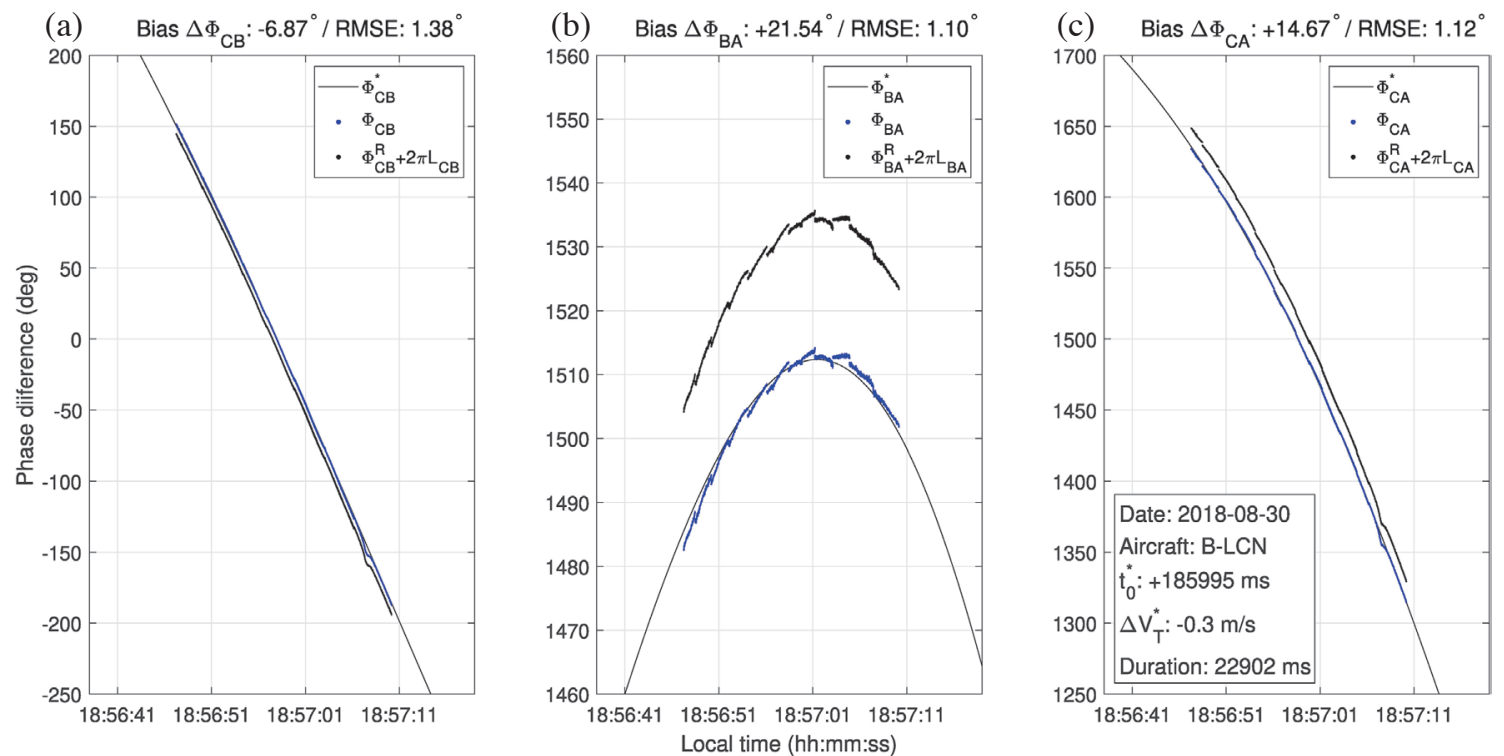

Fig. 7. Comparison between ADS-B-derived phase differences and radar-measured phase differences of the aircraft B-LCN, (a) - (c) illustrate the trend of $\Phi_{C B}, \Phi_{B A}$, and $\Phi_{C A}$, respectively. The duration of radar-measured phase difference is $22902 \mathrm{~ms}$, which is in accordance with the duration of the aircraft inside the main beam of the radar. 

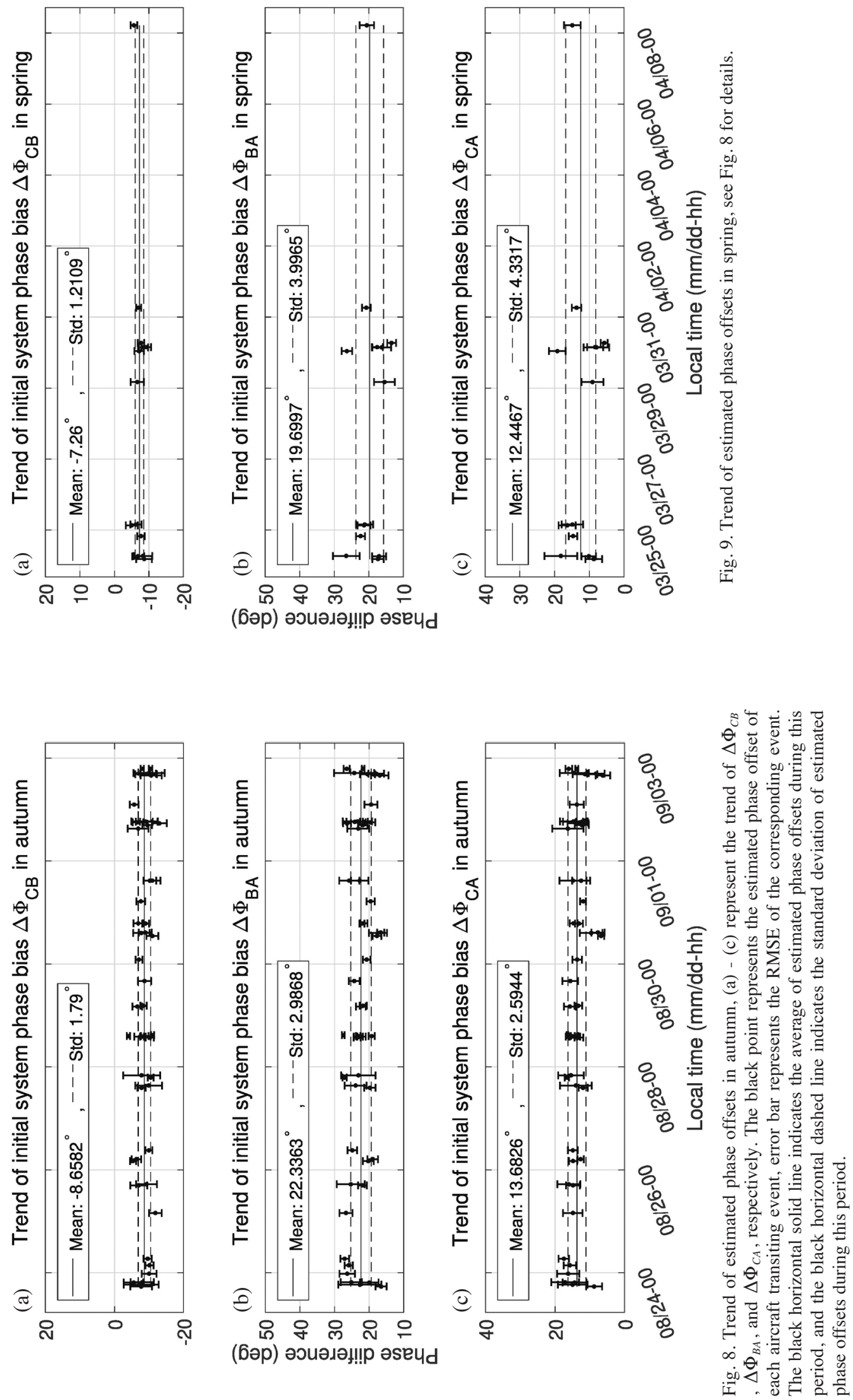
Table 1. Comparisons of system phase biases estimated from different targets.

\begin{tabular}{ccccc}
\hline Target type & Observation duration & $\boldsymbol{\Delta} \boldsymbol{\Phi}_{C B}$ & $\boldsymbol{\Delta} \boldsymbol{\Phi}_{B A}$ & $\boldsymbol{\Delta} \boldsymbol{\Phi}_{C A}$ \\
\hline Commercial aircraft & 23 March 2018 to 8 April 2018 & $-7.26^{\circ} \pm 1.21^{\circ}$ & $+19.70^{\circ} \pm 4.00^{\circ}$ & $+12.45^{\circ} \pm 4.33^{\circ}$ \\
Commercial aircraft & 23 August 2018 to 3 September 2018 & $-8.66^{\circ} \pm 1.79^{\circ}$ & $+22.34^{\circ} \pm 2.99^{\circ}$ & $+13.68^{\circ} \pm 2.59^{\circ}$ \\
Commercial aircraft & 20 December 2018 to 6 January 2019 & $-5.47^{\circ} \pm 1.48^{\circ}$ & $+21.13^{\circ} \pm 3.69^{\circ}$ & $+15.66^{\circ} \pm 3.08^{\circ}$ \\
Multirotor & 25 December 2018 & $-5.37^{\circ} \pm 3.38^{\circ}$ & $+21.31^{\circ} \pm 3.43^{\circ}$ & $+15.94^{\circ} \pm 5.53^{\circ}$ \\
Multirotor & 25 December 2018 & $-5.10^{\circ} \pm 1.21^{\circ}$ & $+21.70^{\circ} \pm 2.54^{\circ}$ & $+16.59^{\circ} \pm 2.11^{\circ}$ \\
FAls & 21 June 2018 to 21 September 2018 & $+6^{\circ}$ & $-1^{\circ}$ & $+5^{\circ}$ \\
FAis & 1 July 2017 to 28 July 2017 & $+13^{\circ}$ & $-4^{\circ}$ & $+9^{\circ}$ \\
FAis & 3 October 2017 to 31 October 2017 & $+7^{\circ}$ & $+2^{\circ}$ & $+9^{\circ}$ \\
\hline
\end{tabular}

mean values of $\Delta \Phi_{C B}, \Delta \Phi_{B A}$, and $\Delta \Phi_{C A}$ of the five experiments made with the aircraft and multirotor are $3.56^{\circ}, 2.64^{\circ}$, and $4.14^{\circ}$, respectively. However, the mean values of the estimated system phase biases from the radar returns of the ionospheric FAIs combined with the IGRF-12 model are substantially different from those of the aircraft/multirotor estimates, which are in the ranges of $+6^{\circ} \sim+13^{\circ}$ for $\Delta \Phi_{C B}$, $-4^{\circ} \sim+2^{\circ}$ for $\Delta \Phi_{B A}$, and $+5^{\circ} \sim+9^{\circ}$ for $\Delta \Phi_{C A}$. The plausible causes that are responsible for the discrepancies in the estimated system phase biases between aircraft/multirotor and FAIs are discussed in next section.

\section{DISCUSSION}

As mentioned in section 1, the phase offsets of the radar system may be attributed to different factors. Some of them may be changed over time, with a time constant ranging from a few seconds to a few years (e.g., electrical characteristics of cables, aging of hardware components, environmental temperature and humidity, timing or clock error, etc.) (Chau et al. 2008), and some of them are independent of time (e.g., placement errors in the antennas). In fact, the phase error caused by the antenna misplacement will result in the error in the angle of arrival of the target echoes (Valentic et al. 1997). Because only the aircraft that are present in the main antenna beam are considered to be employed for the system phase bias estimate, the errors in the angles of arrivals of the aircraft echoes can be treated to be the same and shall be absent in the phase differences between the radar returns received by antenna subarray pair.

On the basis of the comparison between two observation periods in autumn and spring, the differences between $\Delta \Phi_{C B}, \Delta \Phi_{B A}$, and $\Delta \Phi_{C A}$ are all less than $3^{\circ}$. Chau et al. (2014) mentioned that the hardware modification will lead to the notable change in system phase. However, after examining the system log of the Chungli VHF radar, we find that there is no hardware modification during the observation periods. Furthermore, Chen et al. (2002) suggested that the environment temperature play a crucial role in generating system phase error at Chungli VHF radar; therefore, the changes of estimated phase offsets between spring, autumn, and winter might be affected by environmental parameters. According to the weather record at Taoyuan weather station, the average temperature of Taoyuan in spring, autumn, and winter are $20.8,28.1$, and $17.8^{\circ} \mathrm{C}$, respectively. Because of the lack of long-term observations of system phase offsets, the relation between system phase offsets and environment temperatures cannot be verified in this study.

As shown in Table 1, the system phase biases estimated from the FAI echoes are not consistent with those estimated from the aircraft/multirotor echoes. Note that in this study we compare two-dimensional cross correlation functions of the echo intensity distributions in the radar-observed and IGRF-12-simulated echoing regions to estimate the radar system phase offsets. The condition that this method is applicable is the requirement of uniform and symmetric distribution of the echo intensity in the echoing region with respect to the boresight of the antenna beam pattern. However, the occurrence of the 3-m FAIs is unpredictable and random in time and space (Farley et al. 1981; Wang et al. 2011). Therefore, the FAI echo intensity distribution in the expected echoing region will be very likely uneven and asymmetric with respect to the antenna beam boresight if the duration of the radar experiment is not long enough such that FAI echoes do not fill the expected echoing region. As a result, the estimated system phase offset by comparing the cross-correlation function of expected echoing region and that of radar-observed FAI echoing region will be biased. Long term statistics of the ionospheric 3-m Es FAIs observed by the Chungli VHF radar have shown that a uniform and symmetric distribution of the FAI echo intensity in the expected echo region can be achieved as long as the period the data collection is long enough (Wang et al.2011). Therefore, long term observation of the FAIs is needed if the FAI method is employed to estimate unbiased system phase offset.

As shown in Figs. 8 and 9, standard deviations of phase offsets during the whole experiment are all less than $5^{\circ}$. Even though this result is consistent with previous results obtained by other radar groups, a question arises as to 
whether the uncertainty of aircraft position would induce the fluctuation of phase offsets. In order to answer this question, the simulation of the propagation of uncertainty was performed as below.

On the basis of (ICAO 2008), when aircraft fly through nearby the radar, the DVB-T USB dongle not only receives the message of the positions and altitudes of the aircraft, but also receives navigation accuracy category for position (NACp) messages. The NACp block is a 4-bits subfield in 1090ES message, which represents the accuracy level of the aircraft position and altitude. After receiving messages, the NACp subfield shall be decoded as a decimal value from 0 to 11 which is corresponding to different levels of estimated position uncertainty (EPU) and vertical estimated position uncertainty (VEPU). The smaller the NACp values is, the larger the uncertainty of the aircraft position will be. The definition of the EPU is a circle radius that the probability of the reported aircraft position located within this circle centered at the true horizontal position is $95 \%$. Similarly, the VEPU is a 95\% accuracy limit on the vertical position (geometric altitude). For example, for the case of the EPU < $30 \mathrm{~m}$ and $\mathrm{VEPU}<45 \mathrm{~m}$ for $\mathrm{NACp}=9$, there is a $95 \%$ chance that the horizontal distance between the broadcasted position and the true position of the aircraft is less than $30 \mathrm{~m}$, and with the same probability the vertical distance between the broadcasted height and the true height of the aircraft is less than $45 \mathrm{~m}$. It should be noted that the NACp values of all 80 collected flights during the whole experiment are all in the range from 9 to 11, signifying that the values of EPU for all of the broadcasted positions in this experiment are less than $30 \mathrm{~m}$ and those of the VEPU are less than $45 \mathrm{~m}$.

The probability density function of the height distribution of the aircraft can be expressed by a one-dimensional Gaussian function as follows:

$P\left[H_{T}^{\prime}(t)\right]=\frac{1}{\sqrt{2 \pi \sigma_{H}^{2}}} \exp \left\{-\left[H_{T}^{\prime}(t)-H_{T}^{*}(t)\right]^{2} / 2 \sigma_{H}^{2}\right\}$

where $H_{T}^{\prime}(t)$ represents aircraft height, $H_{T}^{*}(t)$ is mean height and $\sigma_{H}$ is a standard deviation. According to the definition of VEPU $<45 \mathrm{~m}$, we can formulate the following expression

$\int_{-45}^{45} \frac{1}{\sigma_{H} \sqrt{2 \pi}} \exp \left\{-h^{2} / 2 \sigma_{H}^{2}\right\} d h=0.95$

Evaluating the definite integral and rearranging the result, we have

$\operatorname{erf}\left(\frac{45}{\sigma_{H} \sqrt{2}}\right)=0.95$

As a result, $\sigma_{H}$ can be given by
$\sigma_{H}=\frac{45}{\sqrt{2} \operatorname{erf}^{-1}(0.95)} \approx 22.96$

This value combined with the horizontal distribution function of the aircraft position will be used in the model to simulate effect of the uncertainty of the 3-dimensional position on the accuracy of the radar system phase bias.

In order to describe the horizontal distribution of aircraft positions, we use a 2-dimensional Gaussian distribution function to model the distribution function of the aircraft position, which is composed of two independent Gaussian distributions with identical standard deviation $\sigma_{r}$ and respective mean $E_{T}^{*}(t)$ and $N_{T}^{*}(t)$ that are given below

$$
\begin{aligned}
& P\left[E_{T}^{\prime}(t)\right]=\frac{1}{\sqrt{2 \pi \sigma_{r}^{2}}} \exp \left\{-\left[E_{T}^{\prime}(t)-E_{T}^{*}(t)\right]^{2} / 2 \sigma_{r}^{2}\right\} \\
& P\left[N_{T}^{\prime}(t)\right]=\frac{1}{\sqrt{2 \pi \sigma_{r}^{2}}} \exp \left\{-\left[N_{T}^{\prime}(t)-N_{T}^{*}(t)\right]^{2} / 2 \sigma_{r}^{2}\right\}
\end{aligned}
$$

where $E_{T}^{\prime}(t)$ and $N_{T}^{\prime}(t)$ represent the horizontal positions of the aircraft in zonal and meridional directions, respectively. It is straightforward to formulate the joint probability density function of $E_{T}^{\prime}(t)$ and $N_{T}^{\prime}(t)$ below

$P\left[E_{T}^{\prime}(t), N_{T}^{\prime}(t)\right]=$
$\frac{1}{\sqrt{2 \pi \sigma_{r}^{2}}} \exp \left(-\left\{\left[E_{T}^{\prime}(t)-E_{T}^{*}(t)\right]^{2}+\left[N_{T}^{\prime}(t)-N_{T}^{*}(t)\right]^{2} / 2 \sigma_{r}^{2}\right\}\right)$

where $P\left[E_{T}^{\prime}(t), N_{T}^{\prime}(t)\right]$ represents the 2-dimensional horizontal distribution of aircraft positions. Performing coordinate transformation from rectangular coordinate to cylindrical coordinate, i.e., $\left[E_{T}^{\prime}(t)-E_{T}^{*}(t)\right]^{2}+\left[N_{T}^{\prime}(t)-N_{T}^{*}(t)\right]^{2}=r^{2}$, and integrating the resulting expression over the whole horizontal plane, we have

$\int_{-\infty}^{+\infty} \int_{-\infty}^{+\infty} P\left[E_{T}^{\prime}(t), N_{T}^{\prime}(t)\right] d E_{T}^{\prime} d N_{T}^{\prime}=$
$\frac{1}{2 \pi \sigma_{r}^{2}} \int_{0}^{2 \pi} \int_{0}^{\infty} \exp \left(-r^{2} / 2 \sigma_{r}^{2}\right) r d r d \theta$

Considering the condition of EPU $<30 \mathrm{~m}$, we can express Eq. (9) as follows:

$\frac{1}{2 \pi \sigma_{r}^{2}} \int_{0}^{2 \pi} \int_{0}^{30} \exp \left(-r^{2} / 2 \sigma_{r}^{2}\right) r d r d \theta=0.95$

Performing definite integral and rearranging the result, we can estimate the value of $\sigma_{r}$ to be $\sigma_{r}=30 / \sqrt{-2 \ln (1-0.95)} \cong 12.26$. Replacing $E_{T}^{*}(t)$ with $E_{T}^{\prime}(t)$ and $N_{T}^{*}(t)$ with $N_{T}^{\prime}(t)$ and $H_{T}^{*}(t)$ with $H_{T}^{\prime}(t)$ in 
Eq. (A9d), we can then rewrite Eqs. (A9d), (A9g), and (5) as follows:

$$
\begin{aligned}
& \vec{R}_{A_{i} T}^{\prime}(t)= \\
& {\left[E_{T}^{\prime}(t)-E_{A_{i}}\right] \hat{e}+\left[N_{T}^{\prime}(t)-N_{A_{i}}\right] \hat{n}+\left[H_{T}^{\prime}(t)-H_{A_{i}}\right] \hat{z}} \\
& \Phi_{i k}^{\prime}(t)=k\left[\left\|\vec{R}_{A_{k} T}^{\prime}(t)\right\|-\left\|\vec{R}_{A_{i} T}^{\prime}(t)\right\|\right] \\
& \Delta \Phi_{i k}^{\prime}=\overline{\Phi_{i k}^{\prime}(t)-\Phi_{i k}^{*}(t)}
\end{aligned}
$$

where $\Delta \Phi_{i k}^{\prime}$ represents the distribution of estimated phase offsets with uncertainty, which will be simulated to compare with the aircraft measurements.

In order to simulate the uncertainty of the estimated system phase bias from aircraft ADS-B signals, we use Gaussian random number generators with zero mean to generate 22902 data points for respective time series of $E_{T}^{\prime}(t)$, $N_{T}^{\prime}(t)$, and $H_{T}^{\prime}(t)$ at $1 \mathrm{~ms}$ time resolution. The standard deviations of the Gaussian functions that we designate for the simulations are 12.26 and $22.96 \mathrm{~m}$ for $E_{T}^{\prime}(t), N_{T}^{\prime}(t)$, and $H_{T}^{\prime}(t)$, respectively. The time period we generate is equals to the duration of the aircraft $\mathrm{B}-\mathrm{LCN}$ inside the main beam, i.e., $22902 \mathrm{~ms}$. Figure 10a shows the probability density distribution of $H_{T}^{\prime}(t)$, and Fig. 10b shows the contour of 2-dimensional probability density distribution of $E_{T}^{\prime}(t)$ and $N_{T}^{\prime}(t)$. These distributions simulate the uncertainty of broadcasted positions of the aircraft for the conditions EPU $<30 \mathrm{~m}$ and
VEPU $<45 \mathrm{~m}$, respectively. Figures $10 \mathrm{c}-\mathrm{e}$ show the histogram of the errors of the estimated phase offsets $\Delta \Phi_{C B}^{\prime}$, $\Delta \Phi_{B A}^{\prime}$, and $\Delta \Phi_{C A}^{\prime}$, respectively. The standard deviation of phase offsets are also estimated based on Gaussian fit are $0.83709^{\circ}, 1.8304^{\circ}$, and $1.8269^{\circ}$, respectively.

These simulation results indicate that the errors of the ADS-B broadcasted positions will significantly influence the accuracy of the estimated phase offsets of the radar system. In this experiment, the uncertainty indicator NACp = 9 implies that the uncertainty of broadcasted positions will contribute about $1^{\circ}$ error in $\Delta \Phi_{C B}$ and about $2^{\circ}$ error in $\Delta \Phi_{B A}$ and $\Delta \Phi_{C A}$. However, observational results show that standard deviations of estimated phase offsets are about $3^{\circ}$ in autumn and about $4^{\circ}$ in spring.

It has been shown that the method of using commercial aircraft equipped with ADS-B system and multirotor can successfully estimate radar system phase bias with some advantages, i.e., reliable, robust, cost-effective, and potentially operational. Nevertheless, the caution should be exercised when we use this method under some special conditions. For example, amplitude saturation of the aircraft echoes that may occur due to too much strong radar returns exceeding receiver dynamic range may increase RMSE of the estimated radar system phase bias, as shown in Fig. 8c. Erroneous time stamp of the aircraft position may be occasionally reported by 1090ES decoding software of the ADSB system due to interference or hardware/software malfunctions, leading to time synchronization problem. Frequent aircraft traffic and high background noise level can increase
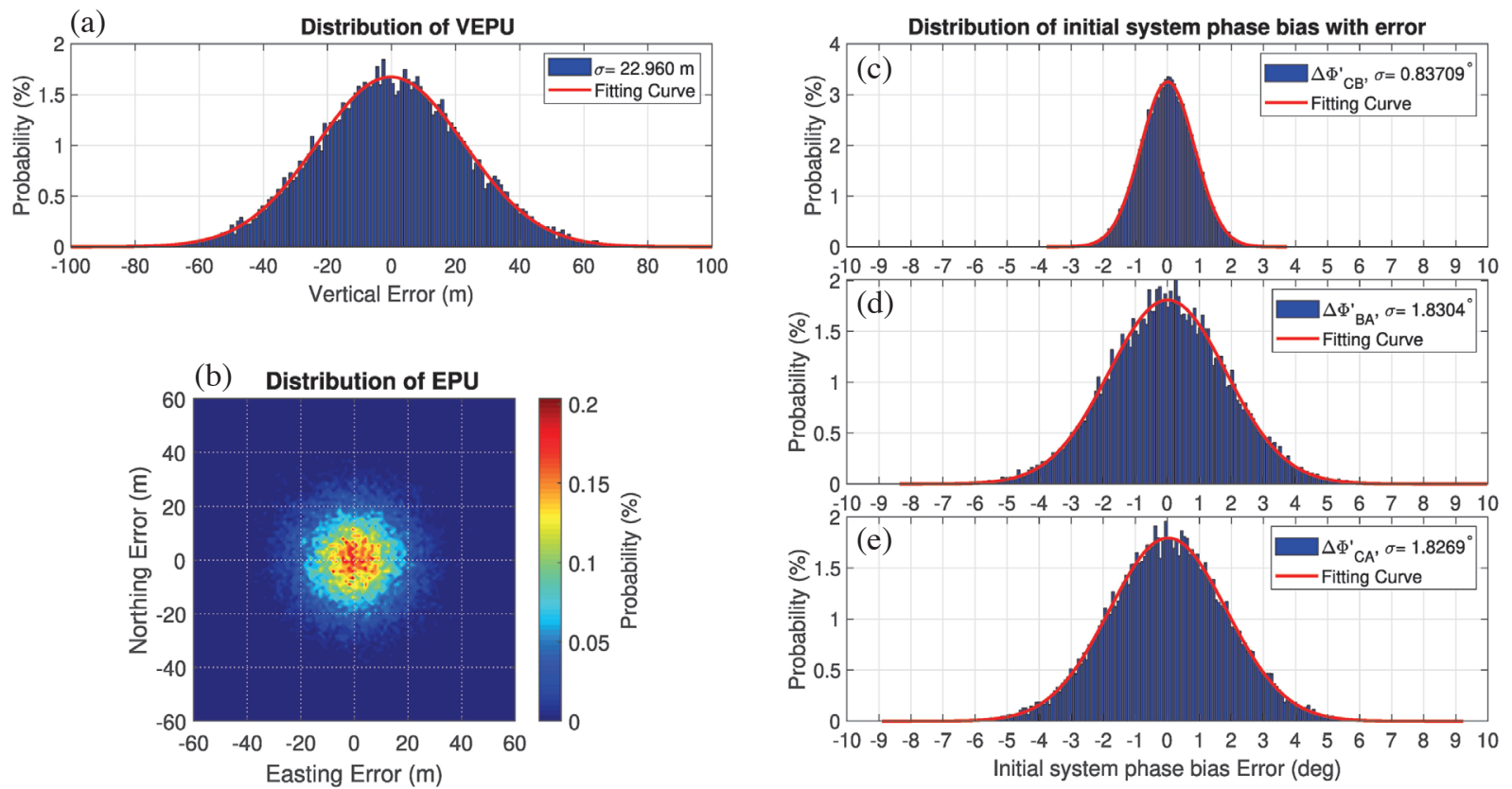

Fig. 10. Simulation of the uncertainty of estimated phase offsets. (a) Histogram of probability density distribution of the error of heights under the condition VEPU $<45 \mathrm{~m}$, the red solid line represents the Gaussian fitting curve of this histogram. (b) 2-dimensional histogram of probability density distribution of the error of horizontal positions under the condition EPU $<30 \mathrm{~m}$. (c) - (e) Histogram of probability density distribution of the error of estimated phase offsets with the corresponding fitting curve. 
decoding failure rate of 1090ES message (Strohmeier et al. 2014). Smaller NACp values (usually, less than 9) may result in unreliable aircraft position reported by ADS-B system and reconstruction of the aircraft trajectory should be greatly cautious.

\section{CONCLUSION}

In this study, we develop an algorithm to use commercial aircraft equipped with ADS-B system and multirotor with GPS facility as radar targets to estimate and calibrate the system phase offsets of the Chungli VHF radar system. From the 80 aircraft transiting events and 2 multirotor experiments, we find that the estimated system phase offsets of the different antenna subarray pairs are very consistent with each other. Namely, the averaged values are $-5.1^{\circ} \sim$ $-8.66^{\circ}$ for $\Delta \Phi_{C B},+19.7^{\circ} \sim+22.34^{\circ}$ for $\Delta \Phi_{B A}$, and $+12.45^{\circ}$ $\sim+16.59^{\circ}$ for $\Delta \Phi_{C A}$, respectively. However, the phase offsets estimated from the FAI echoes, which are $+6^{\circ} \sim+13^{\circ}$ for $\Delta \Phi_{C B},-4^{\circ} \sim+2^{\circ}$ for $\Delta \Phi_{B A}$, and $+5^{\circ} \sim+9^{\circ}$ for $\Delta \Phi_{C A}$, are different from those estimated from the aircraft/multirotor echoes. We believe that the nonuniform and asymmetric distributions of the FAIs in the echoing region with respect to the radar beam boresight direction may be the cause responsible for the inconsistency.

The simulation of the uncertainty of the estimated phase offsets is also performed in this study. The simulation shows that when the uncertainty indicator of the aircraft position NACp is equal to 9 , the uncertainty of ADS-B reported aircraft positions will lead to about $1^{\circ}$ error in $\Delta \Phi_{C B}$ and about $2^{\circ}$ error in $\Delta \Phi_{B A}$ and $\Delta \Phi_{C A}$. This result indicates that the accuracy of the broadcasted position of aircraft is high enough for estimating phase offsets of the radar system. Depending on the high accuracy of this method, the comparison between radar results and other in-situ observations from satellites and rockets can be performed more correctly. In addition to advantages mentioned before, this method still has some other benefits, for example, this method has good adaptability to space weather, in contrast with the method which uses radio stars as targets, and this method might be performed in quasi-real-time after modification.

In this article, the configuration of the ADS-B receiving system and the ADS-B data processing procedures are expressed; moreover, the difficulties and corresponding solutions of the usage of ADS-B data are also described in detail. We expect that this method is helpful to every radar station around the world.

Acknowledgements We would like to thank the surveying team from System Development Center of National ChungShan Institute of Science and Technology and the Taipei Approach of Air Navigation and Weather Services, Civil Aeronautics Administration, MOTC in Taiwan, for their contributions toward the work presented in this article.

\section{REFERENCES}

Aso, T., T. Tsuda, and S. Kato, 1979: Meteor radar observations at Kyoto University. J. Atmos. Terr. Phys., 41, 517-525, doi: 10.1016/0021-9169(79)90075-8. [Link]

Balsley, B. B. and K. S. Gage, 1980: The MST radar technique: Potential for middle atmospheric studies. Pure Appl. Geophys., 118, 452-493, doi: 10.1007/ BF01586464. [Link]

Chau, J. L., D. L. Hysell, K. M. Kuyeng, and F. R. Galindo, 2008: Phase calibration approaches for radar interferometry and imaging configurations: Equatorial spread F results. Ann. Geophys., 26, 2333-2343, doi: 10.5194/ angeo-26-2333-2008. [Link]

Chau, J. L., T. Renkwitz, G. Stober, and R. Latteck, 2014: MAARSY multiple receiver phase calibration using radio sources. J. Atmos. Sol.-Terr. Phys., 118, 55-63, doi: 10.1016/j.jastp.2013.04.004. [Link]

Chen, J.-S., J. Röttger, and Y.-H. Chu, 2002: System phase calibration of VHF spaced antennas using the echoes of aircraft and incorporating the frequency domain interferometry technique. Radio Sci., 37, 13-1-13-13, doi: 10.1029/2002RS002604. [Link]

Chen, J.-S., C.-L. Su, Y.-H. Chu, G. Hassenpflug, and M. Zecha, 2009: Extended Application of a Novel Phase Calibration Approach of Multiple-Frequency Range Imaging to the Chung-Li and MU VHF Radars. J. Atmos. Ocean. Technol., 26, 2488-2500, doi: 10.1175/2009JTECHA1295.1. [Link]

Chen, J.-S., S.-C. Tsai, C.-L. Su, and Y.-H. Chu, 2016: Evaluation of multifrequency range imaging technique implemented on the Chung-Li VHF atmospheric radar. Atmos. Meas. Tech., 9, 2345-2355, doi: 10.5194/amt9-2345-2016. [Link]

Chu, Y.-H. and C.-Y. Wang, 1997: Interferometry observations of three-dimensional spatial structures of sporadic $E$ irregularities using the Chung-Li VHF radar. $R a$ dio Sci., 32, 817-832, doi: 10.1029/96RS03578. [Link]

Chu, Y.-H., L.-P. Chian, and C.-H. Liu, 1991: The investigations of the atmospheric precipitations by using Chung-Li VHF radar. Radio Sci., 26, 717-729, doi: 10.1029/91RS00830. [Link]

Clark, R.R., 1978: Meteor wind data for global comparisons. J. Atmos. Terr. Phys., 40, 905-911, doi: 10.1016/00219169(78)90141-1. [Link]

Farley, D. T., 1985: Theory of equatorial electrojet plasma waves: New developments and current status. $J$. Atmos. Terr. Phys., 47, 729-744, doi: 10.1016/00219169(85)90050-9. [Link]

Farley, D. T., H. M. Ierkic, and B. G. Fejer, 1981: Radar interferometry: A new technique for studying plasma turbulence in the ionosphere. J. Geophys. Res., 86, 1467-1472, doi: 10.1029/JA086iA03p01467. [Link]

Glanz, F. H., 1971: Azimuth Measuring System for a 
Meteor-Trails Radar.IEEE Transactions on Geoscience Electronics, 9, 56-62, doi: 10.1109/TGE.1971.271461. [Link]

Hager, J. W., J. F. Behensky, and B. W. Drew, 1989: The Universal Grids: Universal Transverse Mercator (UTM) and Universal Polar Stereographic (UPS). DMA Technical Manual, DMATM 8358.2, Defense Mapping Agency Hydrographic/Topographic Center, $49 \mathrm{pp}$.

Holdsworth, D. A., M. Tsutsumi, I. M. Reid, T. Nakamura, and T. Tsuda, 2004: Interferometric meteor radar phase calibration using meteor echoes. Radio Sci., 39, 1-12, doi: 10.1029/2003RS003026. [Link]

ICAO, 2008: Technical Provisions for Mode S Services and Extended Squitter, 9871-AN/464 First Edition., ICAO, Montréal, QC, Canada, 230 pp.

Kuong, R.-M., Y.-H. Chu, S.-Y. Su, and C.-L. Su, 2003: The Chung-Li VHF radar as a meteor radar: System phase bias estimation and experimental results. Terr. Atmos. Ocean. Sci., 14, 113-132, doi: 10.3319/ TAO.2003.14.2.113(A). [Link]

Li, G., B. Ning, Y.-H. Chu, I. M. Reid, L. Hu, B. K. Dolman, J. Xiong, G. Jiang, G. Yang, and C. Yan, 2014: Structural evolution of long-duration meteor trail irregularities driven by neutral wind. J. Geophys. Res., 119, 10348-10357, doi: 10.1002/2014JA020116. [Link]

Lin, F. F., C. Y. Wang, C. L. Su, K. Shiokawa, S. Saito, and Y. H. Chu, 2016: Coordinated observations of $\mathrm{F}$ region $3 \mathrm{~m}$ field-aligned plasma irregularities associated with medium-scale traveling ionospheric disturbances. J. Geophys. Res., 121, 3750-3766, doi: 10.1002/2016JA022511. [Link]

NGA, 2014: The Universal Grids and the Transverse Mercator and Polar Stereographic Map Projections. Report NGA.SIG.0012_2.0.0_UTMUPS, National Geospatial-Intelligence Agency, $86 \mathrm{pp}$.

Palmer, R. D., S. Vangal, M. F. Larsen, S. Fukao, T. Nakamura, and M. Yamamoto, 1996: Phase calibration of VHF spatial interferometry radars using stellar sources. Radio Sci., 31, 147-156, doi: 10.1029/95RS02319. [Link]

Providakes, J. P., W. E. Swartz, D. T. Farley, and B. G. Fejer, 1983: First VHF auroral radar interferometer observations. Geophys. Res. Lett., 10, 401-404, doi: 10.1029/GL010i005p00401. [Link]

Rapp, M. and F.-J. Lübken, 2004: Polar mesosphere summer echoes (PMSE): Review of observations and current understanding. Atmos. Chem. Phys., 4, 2601-2633, doi: 10.5194/acp-4-2601-2004. [Link]

Robertson, D. S., D. T. Liddy, and W. G. Elford, 1953: Measurements of winds in the upper atmosphere by means of drifting meteor trails I. J. Atmos. Terr. Phys., 4, 255270, doi: 10.1016/0021-9169(53)90059-2. [Link]

Saito, S., M. Yamamoto, H. Hashiguchi, A. Maegawa, and
A. Saito, 2007: Observational evidence of coupling between quasi-periodic echoes and medium scale traveling ionospheric disturbances. Ann. Geophys., 25, 2185-2194, doi: 10.5194/angeo-25-2185-2007. [Link]

Schlatter, N. M., T. Grydeland, N. Ivchenko, V. Belyey, J. Sullivan, C. La Hoz, and M. Blixt, 2013: Radar interferometer calibration of the EISCAT Svalbard Radar and a additional receiver station. J.Atmos. Sol.-Terr. Phys., 105-106, 287-292, doi: 10.1016/j.jastp.2012.11.017. [Link]

Stewart, R. W., L. Crockett, D. Atkinson, K. Barlee, D. Crawford, I. Chalmers, M. Mclernon, and E. Sozer, 2015: A low-cost desktop software defined radio design environment using MATLAB, simulink, and the RTL-SDR. IEEE Comm. Mag., 53, 64-71, doi: 10.1109/MCOM.2015.7263347. [Link]

Strohmeier, M., M. Schafer, V. Lenders, and I. Martinovic, 2014: Realities and challenges of nextgen air traffic management: The case of ADS-B. IEEE Comm. Mag., 52, 111-118, doi: 10.1109/MCOM.2014.6815901. [Link]

Su, C. L., H. C. Chen, Y. H. Chu, M. Z. Chung, R. M. Kuong, T. H. Lin, K. J. Tzeng, C. Y. Wang, K. H. $\mathrm{Wu}$, and K. F. Yang, 2014: Meteor radar wind over Chung-Li $\left(24.9^{\circ} \mathrm{N}, 121^{\circ} \mathrm{E}\right)$, Taiwan, for the period 10-25 November 2012 which includes Leonid meteor shower: Comparison with empirical model and satellite measurements. Radio Sci., 49, 597-615, doi: 10.1002/2013RS005273. [Link]

Sullivan, J. M., N. Ivchenko, M. Lockwood, T. Grydeland, E. M. Blixt, and B. S. Lanchester, 2006: Phase calibration of the EISCAT Svalbard Radar interferometer using optical satellite signatures. Ann. Geophys., 24, 2419-2427, doi: 10.5194/angeo-24-2419-2006. [Link]

Tsai, S.-C., J.-S. Chen, Y.-H. Chu, C.-L. Su, and J.-H. Chen, 2018: High-range resolution spectral analysis of precipitation through range imaging of the Chung-Li VHF radar. Atmos. Meas. Tech., 11, 581-592, doi: 10.5194/ amt-11-581-2018. [Link]

Valentic, T. A., J. P. Avery, S. K. Avery, and R. C. Livingston, 1997: Self-survey calibration of meteor radar antenna arrays. IEEE Trans. Geosci. Remote Sensing, 35, 524-531, doi: 10.1109/36.581960. [Link]

Vandepeer, B. G. W. and I. M. Reid, 1995: Some preliminary results obtained with the new Adelaide MF Doppler radar. Radio Sci., 30, 1191-1203, doi: 10.1029/95RS00732. [Link]

Vidal, L., 2013: ADS-B task force - KOLKATA, ADS-B OUT \& IN - AIRBUS STATUS, Report, AIRBUS S.A.S, 36 pp.

Wang, C.-Y., 1999: The observations of ionospheric sporadic E irregularities using the Chungli VHF radar. Ph.D. Thesis, National Central University, Taoyuan City, Taiwan, 162 pp. 
Wang, C.-Y. and Y.-H. Chu, 2001: Interferometry investigations of blob-like sporadic $\mathrm{E}$ plasma irregularity using the Chung-Li VHF radar. J. Atmos. Sol.-Terr. Phys., 63, 123-133, doi: 10.1016/S1364-6826(00)00141-3. [Link]

Wang, C.-Y., Y.-H. Chu, C.-L. Su, R.-M. Kuong, H.-C. Chen, and K.-F. Yang, 2011: Statistical investigations of layer-type and clump-type plasma structures of 3-m field-aligned irregularities in nighttime sporadic E region made with Chung-Li VHF radar. J. Geophys. Res., 116, A12311, doi: 10.1029/2011JA016696. [Link]

Yu, T.-Y. and R. D. Palmer, 2001: Atmospheric radar imaging using multiple-receiver and multiple-frequency techniques. Radio Sci., 36, 1493-1503, doi: 10.1029/2000RS002622. [Link]

\section{APPENDIX}

\section{Appendix 1. Coordinate Transformation from WGS84 to Mercator Coordinate}

On the basis of the conversion algorithms published by Hager et al. (1989) and NGA (2014), equations for converting aircraft positions from geographic longitude-latitude pairs $\left(\lambda_{i}, \phi_{i}\right)$ to transverse Mercator projection eastingnorthing pairs $\left(E_{i}, N_{i}\right)$ are given below:

$\cos \chi_{i}=\frac{2 \cos \phi_{i}}{\left(1+\sin \phi_{i}\right) / P_{i}+\left(1-\sin \phi_{i}\right) P_{i}}$

$\sin \chi_{i}=\frac{\left(1+\sin \phi_{i}\right) / P_{i}-\left(1-\sin \phi_{i}\right) P_{i}}{\left(1+\sin \phi_{i}\right) / P_{i}+\left(1-\sin \phi_{i}\right) P_{i}}$

$P_{i}=\exp \left[e \tanh ^{-1}\left(e \sin \phi_{i}\right)\right]$

$u_{i}=\tanh ^{-1}\left[\cos \left(\chi_{i}\right) \sin \left(\lambda_{i}-\lambda_{o}\right)\right]$

$v_{i}=a \tan 2\left[\sin \left(\chi_{i}\right), \cos \left(\chi_{i}\right) \cos \left(\lambda_{i}-\lambda_{o}\right)\right]$

$E_{i}=k_{o} R_{4}\left[u_{i}+\sum_{n=1}^{6} a_{2 n} \sinh \left(2 n u_{i}\right) \cos \left(2 n v_{i}\right)\right]+E_{o}$

$N_{i}=k_{o} R_{4}\left[v_{i}+\sum_{n=1}^{6} a_{2 n} \cosh \left(2 n u_{i}\right) \sin \left(2 n v_{i}\right)\right]+N_{o}$

where $e$ is the eccentricity, $R_{4}$ represents the meridional isoperimetric radius, $\lambda_{o}$ represents the central meridian, $k_{o}$ is the central scale factor, $E_{o}$ and $N_{o}$ represent the central meridian easting and the equator northing, respectively. In particular, constants $e, R_{4}, a_{2}, a_{4}, \ldots, a_{12}$ all depend on the choice of the reference ellipsoid, and constants $\lambda_{o}, k_{o}, E_{o}$, and $N_{o}$ depend on the local map projection chosen in this experiment. Therefore, constants for converting aircraft positions from WGS84 coordinate to TM2 coordinate can be determined as shown in Table A1.

For the purpose of determining the accuracy of converted positions, a computational accuracy of this conversion algorithm is required. NGA (2014) introduced a quantity called index $\delta$ as an indicator of computational error bound of geographic longitude-latitude pairs $(\lambda, \phi)$. The quantity $\delta_{i}$ is given by

$\delta_{i}=$

$\operatorname{Minimum}\left(\left|\lambda_{i}-\lambda_{o}\right|,\left|\lambda_{i}-\lambda_{o}-\pi\right|,\left|\lambda_{i}-\lambda_{o}+\pi\right|, \frac{\pi}{2}-\phi_{i}, \frac{\pi}{2}+\phi_{i}\right)$

In this experiment, all of the aircraft that transit across the boresight of the radar beam are very close to the chosen central meridian of TM2 coordinate. As a result, all of $\delta_{i}$ values are less than $0.5^{\circ}$, leading to that the distances between $\left(\lambda_{i}, \phi_{i}\right)$ and $\left(E_{i}, N_{i}\right)$ are all less than $10^{-9} \mathrm{~m}$ (NGA 2014).

\section{Appendix 2. Reconstruction of Aircraft Trajectory}

Before performing the time synchronization, the trajectory of the aircraft should be reconstructed first. To begin with, on the basis of the linear fitting method, the heading direction of the aircraft was retrieved from the eastingnorthing pairs $\left(E_{i}, N_{i}\right)$ of the aircraft positions obtained via Eqs. (A1f) and (A1g) in accordance with the following expression:

$$
\begin{aligned}
& \psi_{T}= \operatorname{sgn}\left(E_{n}-E_{1}\right) \\
& \cdot \operatorname{atan} 2\left[n \sum_{i=1}^{n} E_{i}^{2}-\left(\sum_{i=1}^{n} E_{i}\right)^{2}, \operatorname{sgn}\left(E_{n}-E_{1}\right)\right. \\
&\left.\cdot\left(n \sum_{i=1}^{n} E_{i} N_{i}-\sum_{i=1}^{n} E_{i} \sum_{i=1}^{n} N_{i}\right)\right]
\end{aligned}
$$

where $\psi_{T}$ represents the heading of the aircraft which is defined as the angle clockwise from geographic north, $n$ is the number of the data points of the reported aircraft positions employed for regression line fitting. Then, three dimensional TM2 position sets of the aircraft trajectory can be reconstructed as a function of time as follows:

$\hat{v}_{T}=\sin \left(\psi_{T}\right) \hat{e}+\cos \left(\psi_{T}\right) \hat{n}$

$E_{T}\left(t, t_{0}, \Delta V_{T}\right)=E_{\text {init }}+\left(t+t_{0}\right) \cdot\left(V_{T}+\Delta V_{T}\right) \hat{v}_{T} \cdot \hat{e}$

$N_{T}\left(t, t_{0}, \Delta V_{T}\right)=N_{\text {init }}+\left(t+t_{0}\right) \cdot\left(V_{T}+\Delta V_{T}\right) \hat{v}_{T} \cdot \hat{n}$

$H_{T}\left(t, t_{0}, \Delta V_{T}\right)=H_{\text {init }}+\left(t+t_{0}\right) \cdot\left(V_{T}+\Delta V_{T}\right) \hat{v}_{T} \cdot \hat{z}$

where $V_{T}$ is the broadcasted ground speed of the aircraft, $E_{\text {init }}$, $N_{\text {init }}, H_{\text {init }}$ are the initial easting, northing and height of the aircraft, respectively, $\hat{v}_{T}$ is the unit vector of the heading of 
the aircraft, $\hat{z}$ is the upward unit vector, $\hat{e}$ and $\hat{n}$ represent the unit vector of TM2 easting and northing, respectively. $\Delta V_{T}$ is the correction for calibrating the possible bias of broadcasted speed of the aircraft, and $t_{0}$ represents the correction for time synchronization as mentioned above. Note that the aircraft selected for the radar system phase bias estimate should fly at a steady velocity and at a fixed altitude. From Eqs. (A5a), (A5b), and (A5c), we can obtain the flight information on the aircraft, including distance vectors from each center of subarrays $A_{i}$ to the aircraft $\vec{R}_{A_{i} T}$, radial velocity $V_{A_{i} T}$, direction in azimuth $\phi_{A_{i} T}$, direction in elevation $\theta_{A_{i} T}$, and the phase difference between two subarrays $\Phi_{i k}$ are derived as follows:

$$
\begin{aligned}
& \vec{R}_{A_{i} T}\left(t, t_{0}, \Delta V_{T}\right)= \\
& {\left[E_{T}\left(t, t_{0}, \Delta V_{T}\right)-E_{A_{i}}\right] \hat{e}+\left[N_{T}\left(t, t_{0}, \Delta V_{T}\right)-N_{A_{i}}\right] \hat{n}+} \\
& {\left[H_{T}\left(t, t_{0}, \Delta V_{T}\right)-H_{A_{i}}\right] \hat{z}} \\
& \hat{r}_{A_{i} T}\left(t, t_{0}, \Delta V_{T}\right)=\frac{\vec{R}_{A_{i} T}\left(t, t_{0}, \Delta V_{T}\right)}{\left\|\vec{R}_{A_{i} T}\left(t, t_{0}, \Delta V_{T}\right)\right\|} \\
& V_{A_{i} T}\left(t, t_{0}, \Delta V_{T}\right)=\left(V_{T}+\Delta V_{T}\right) \hat{v}_{T} \cdot \hat{r}_{A_{i} T}\left(t, t_{0}, \Delta V_{T}\right) \\
& \phi_{A_{i} T}\left(t, t_{0}, \Delta V_{T}\right)= \\
& \operatorname{atan} 2\left[\vec{R}_{A_{i} T}\left(t, t_{0}, \Delta V_{T}\right) \cdot \hat{e}, \vec{R}_{A_{i} T}\left(t, t_{0}, \Delta V_{T}\right) \cdot \hat{n}\right] \\
& \theta_{A_{i} T}\left(t, t_{0}, \Delta V_{T}\right)=\sin ^{-1}\left[\frac{\vec{R}_{A_{i} T}\left(t, t_{0}, \Delta V_{T}\right) \cdot \hat{z}}{\left.\left\|\vec{R}_{A_{i} T}\left(t, t_{0}, \Delta V_{T}\right)\right\|\right]}\right. \\
& \Phi_{i k}\left(t, t_{0}, \Delta V_{T}\right)= \\
& k\left[\left\|\vec{R}_{A_{i} T}\left(t, t_{0}, \Delta V_{T}\right)\right\|-\left\|\vec{R}_{A_{i} T}\left(t, t_{0}, \Delta V_{T}\right)\right\|\right]
\end{aligned}
$$

where $k$ is wavenumber, $\left(E_{A_{i}}, N_{A_{i}}, H_{A_{i}}\right)$ represents the TM2 position of each subarray $A_{i}$. With these crucial flight parameters, the time synchronization between aircraft and craft can be achieved.

\section{Appendix 3. Time Synchronization}

Note that there are two ways to obtain the radial velocity of the aircraft. One is to calculate the phase change of the radar returns from the aircraft in accordance with Eq. (4). The other one is to analyze the aviation messages broadcasted by the ADS-B avionics system based on equations from Eqs. (A3) - (A7b). In light of the fact that there is timing discrepancy between Chungli VHF radar and the aircraft ADS-B system, time synchronization should be performed when the data collected by the latter are employed to estimate the system phase bias of the former. In this study, we compare the time sequences of the radial velocities estimated from the radar echoes and the ADS-B broadcasted signal to estimate the timing difference $t_{0}$ between these two systems. In addition to $t_{0}$, we note that the reported aircraft flight speed error $\Delta V_{T}$ broadcasted by the ADS-B system can also contribute to timing discrepancy between radar and aircraft. In this study, we acquire the estimated timing difference $t_{0}^{*}$ and $\Delta V_{T}^{*}$ by finding the arguments of the minimum of the root-mean-square error (RMSE) of the function $V_{A_{i} T}^{R}(t)-V_{A_{i} T}\left(t, t_{0}, \Delta V_{T}\right)$ that is given by

$$
t_{0}^{*}, \Delta V_{T}^{*}=\underset{t_{0}, \Delta V_{T}}{\operatorname{argmin}}\left(\sqrt{E\left\{\left[V_{A_{i} T}^{R}(t)-V_{A_{i} T}\left(t, t_{0}, \Delta V_{T}\right)\right]^{2}\right\}}\right)
$$

where $V_{A_{i}}^{R}(t)$ and $V_{A_{i} T}\left(t, t_{0}, \Delta V_{T}\right)$ are, respectively, the Doppler (radial) velocities of the radar echoes and the ADS-B broadcasted ground speed. It is worthy to point out that, theoretically, the corrections $t_{0}^{*}$ and $\Delta V_{T}^{*}$ may have tiny differences for different spaced antenna subarrays $A_{i}$. However, the differences can be ignored due to small separations between the subarrays for the Chungli VHF radar. Figure A1 compares time sequences of the radial velocities of the aircraft before and after time synchronization for the aircraft B-LCN on 30 August 2018. As shown in Fig. 7, the estimated corrections $t_{0}^{*}$ in time and $\Delta V_{T}^{*}$ in radial velocity are $+185.995 \mathrm{~s}$ and $-0.3 \mathrm{~m} \mathrm{~s}^{-1}$, respectively, with a corresponding RMSE of $0.25079 \mathrm{~m} \mathrm{~s}^{-1}$ after performing the time synchronization for $V_{A_{i} T}\left(t, t_{0}, \Delta V_{T}\right)$.

After the time synchronization, Eqs. (A5a), (A5b), (A5c), (A6), (A7c), (A7d), and (A7e) can be rewritten as follows:

$E_{T}^{*}(t)=E_{\text {init }}+\left(t+t_{0}^{*}\right) \cdot\left(V_{T}+\Delta V_{T}^{*}\right) \hat{v}_{T} \cdot \hat{e}$

$N_{T}^{*}(t)=N_{\text {init }}+\left(t+t_{0}^{*}\right) \cdot\left(V_{T}+\Delta V_{T}^{*}\right) \hat{v}_{T} \cdot \hat{n}$

$H_{T}^{*}(t)=H_{\text {init }}+\left(t+t_{0}^{*}\right) \cdot\left(V_{T}+\Delta V_{T}^{*}\right) \hat{v}_{T} \cdot \hat{z}$

$\vec{R}_{A, T}^{*}(t)=$

$\left[E_{T}^{*}(t)-E_{A_{i}}\right] \hat{e}+\left[N_{T}^{*}(t)-N_{A_{i}}\right] \hat{n}+\left[H_{T}^{*}(t)-H_{A_{i}}\right] \hat{z}$

$\phi_{A_{i} T}^{*}(t)=\operatorname{atan} 2\left[\vec{R}_{A_{i} T}^{*}(t) \cdot \hat{e}, \vec{R}_{A_{i} T}^{*}(t) \cdot \hat{n}\right]$

$\theta_{A_{i} T}^{*}(t)=\sin ^{-1}\left[\frac{\vec{R}_{A_{i} T}^{*}(t) \cdot \hat{z}}{\left\|\vec{R}_{A_{i} T}^{*}(t)\right\|}\right]$

$\Phi_{i k}^{*}(t)=k\left[\left\|\vec{R}_{A_{k} T}^{*}(t)\right\|-\left\|\vec{R}_{A_{i} T}^{*}(t)\right\|\right]$ 
Equations (A9a) - (A9g) represent the synchronized quantities of the aircraft. Note that only quantities inside the main beam were collected for estimating phase offsets. According to calculations of Eqs. (A9e) and (A9f), the synchronized elevation and azimuth angle as well as the transiting path of the aircraft with respect to the radar can be obtained; therefore, the actual path of the aircraft which was inside the main beam can be determined. Moreover, the actual phase difference pairs $\Phi_{i k}^{*}(t)$ inside the main beam were also determined based on Eq. (A9g).

Table A1. Coefficients of Eqs. (A1a) to (A1g) and (A2) for converting aircraft coordinate from WGS84 to TM2.

\begin{tabular}{cc}
\hline WGS84 constants: & TM2 constants: \\
\hline$e: 0.08181919084262149433$ & $k_{o}: 0.9999$ \\
$R_{4}: 6367449.1458234153093 \mathrm{~m}$ & $E_{o}: 250000 \mathrm{~m}$ \\
$a_{2}: 8.3773182062446983032 \times 10^{-4}$ & $\lambda_{o}: 121^{\circ}$ \\
$a_{4}: 7.608527773572489156 \times 10^{-7}$ & $N_{o}: 0 \mathrm{~m}$ \\
$a_{6}: 1.19764550324249210 \times 10^{-9}$ & \\
$a_{8}: 2.4291706803973131 \times 10^{-12}$ & \\
$a_{10}: 5.711818369154105 \times 10^{-15}$ & \\
$a_{12}: 1.47999802705262 \times 10^{-17}$ & \\
\hline
\end{tabular}

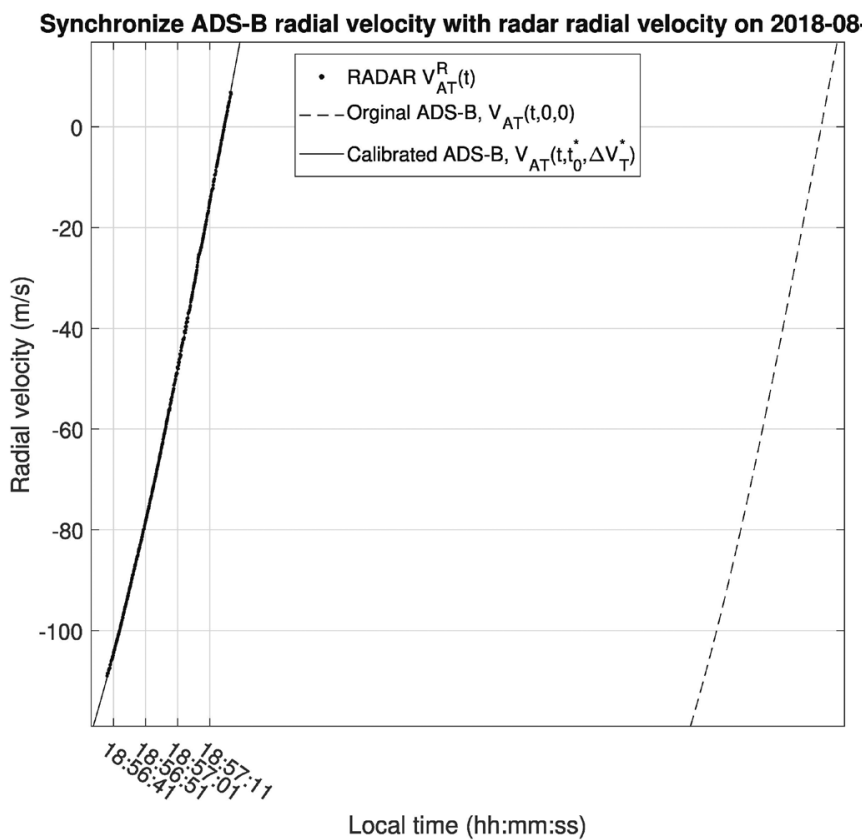

Fig. A1. Comparison between the trend of estimated and observed radial velocities of the aircraft B-LCN. Note that all three radial velocities are with respect to the subarray A. 